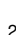

3

\title{
Stability Constrains how Populations Spread Risk in a Model of Food Exchange
}

James P. O'Dwyer ${ }^{12 *}$

\section{Summary}

Exchange of food between human populations can mitigate the risk arising from environmental variability and consequent variability in local food production. The precise network, or mode of exchange varies according to context, but these modes of exchange tend to fall into a handful of categories: specifically, giving without expectation of receiving, reciprocal relationships, and pooling. In this manuscript we address whether the stability of populations to small perturbations can play a role in understanding why only a limited number of modes of exchange are typical. We develop a simple model of exchange between populations located in different environments, and show that any mode of exchange in our model will help to buffer the risk associated with local environmental variability. However, we find only a limited set of modes of exchange will guarantee population stability when resources are scarce, and these mirror the empirical classifications of exchange. We don't consider directly the evolution of this cooperative behavior, but our results demonstrate that stability may provide a filter for viable modes of exchange once cooperation has arisen.

Keywords: exchange | trade | environmental variability | coupled human-natural systems

\footnotetext{
${ }^{1}$ Department of Plant Biology, University of Illinois, Urbana, IL 61801, USA

${ }^{2}$ Carl R. Woese Institute for Genomic Biology, University of Illinois, Urbana, IL 61801, USA

*Correspondence: jodwyer@illinois.edu
} 


\section{Introduction}

Human survival depends to a large extent on the reliable availability of food. For sedentary ancestral cultures relying on agriculture, much of this caloric requirement would be supplied by the cultivation of domesticated plants grown locally. Groups of individuals relying on this mode of provisioning are therefore potentially vulnerable to variation in their local environment, given that the productivity of crops can vary significantly from year to year and across relatively small spatial separations. ${ }^{1,2}$ Meanwhile, social interactions offer the potential to buffer this variability, via the exchange of resources between groups of individuals living in distinct environments. ${ }^{3-9}$

Spreading of risk intuitively offers the potential to mitigate the effects of a variable environment, and is observed across many human cultures and contexts. ${ }^{10-12}$ There is also evidence that exchange of crops in particular has occurred over large enough scales to buffer the risk of local productivity variation. Within the American Southwest, there is extensive work establishing that the agricultural niche (primarily indicating the ability to grow maize) is limited by climate, and has been highly variable through time $^{2}$ and across space ${ }^{13}$ on length-scales of $10 \mathrm{~s}$ of $\mathrm{km}$. In parallel, there is work in this same system establishing that maize has very likely been exchanged on length-scales of up to $100 \mathrm{~km} .{ }^{14-17}$ In summary, when the conditions suitable for agricultural success are sufficiently variable over space and time, it is precisely this kind of volatile environment that offers the opportunity for gains to be made via transfer of food.

More generally, multiple, distinct modes of food exchange have been documented, with a pioneering classification developed by Sahlins. ${ }^{3}$ When interactions are positive, these classic categories reduce to (i) generalized reciprocity, where resources are given without the expectation of a return, (ii) balanced reciprocity, where exchange is equal in each direction between a pair of partners, and (iii) pooling, where resources are given to a central hub and then redistributed. There has been extensive analytical and empirical work in the human behavioral ecology of food exchange since Sahlins' analysis, $5,7,8,17-21$ meaning that this early classification is unlikely to be comprehensive, and any one typology is unlikely to hold across all examples and scales of food exchange. On the other hand, this kind of classification raises the very natural question of whether some subset of all possible modes of food exchange do exhibit features that allow them to persist in the longer-term.

Here we address this open question using a novel approach based on the analysis of stability. We develop a model based on the consumption and depletion of a natural resource by multiple groups in different locations, drawing from existing consumer-resource models in ecology. ${ }^{22-29}$ We first show that any mode of exchange can buffer a range of types of environmental variation, so long as this variation is uncorrelated across locations. Importantly, this spreading of risk doesn't just reduce the variation in the total system size, summed across all sites, ${ }^{30}$ but guarantees reduced variation relative to mean population sizes at each individual site. In contrast, exchange cannot buffer variability in cases of system-wide changes in productivity, for example during an extended drought. ${ }^{31-33}$ However, we find that the situation during system-wide scarcity can be even worse than just reduced population sizes. For sufficiently low productivity we show that generic modes of exchange, which we term non-stabilizing, will lead to instability, and potential depopulation. We also find that only a handful of cases, which we term stabilizing modes of exchange, guarantee stable population sizes even during scarcity. These include Sahlins' categories of generalized reciprocity, balanced reciprocity, and pooling, alongside more general, decentralized modes of redistribution.

\section{Uncertainty and Exchange}

For humans relying on agriculture or foraging for caloric intake, uncertainty mediated by the weather is a fact of life. Variability in productivity from year to year has been a constant, identified and often supported via climate reconstruction across multiple ancestral systems, ${ }^{2,13,34-38}$ documented in present day societies, ${ }^{37,39,40}$ and with important implications for future food security. ${ }^{41-43}$ As one might expect for such 
an inevitable challenge, there are multiple, established strategies that human populations have used to mitigate, or buffer the effects of changing productivity. These have previously been classified as ${ }^{37,44,45}$

- Diversification. Broadening the caloric base, for example via a broader range of farmed locations, or a broader range of plant (or animal) species, or even just a broader range of strains of the same crop planted in the same year, with different expected yields and levels of resilience to weather variability ${ }^{46,47}$

- Storage. The storage of food from good periods for consumption in times of scarcity, usually limited by technology (e.g. subterranean storage of maize has been proposed (and disputed) as a buffering mechanism for Mississippian societies ${ }^{48}$ )

- Mobility. The choice to move on from a region experiencing lower productivity-likely more limited (though still possible) for farming crops than e.g. hunter-gatherers, and perhaps mediated via exchange of information over long-distances in order to determine new, more productive locations with some degree of certainty. ${ }^{37}$

- Exchange. A fourth mechanism to mitigate uncertainty is the exchange of food (and/or other goods) with other individuals or populations. ${ }^{9}$ If the yield for a particular household or population is at least somewhat uncorrelated with its exchange partners, there is the potential that by linking and transferring food between locations, variability across location can smooth variability in productivity over time in a given location. In addition to this positive form of exchange, this fourth category has also been thought to incorporate more 'negative' forms of transfer, including theft and appropriation of food from other locations. $^{3}$

We will focus here on food exchange as a mechanism to buffer uncertainty in agricultural productivity, and will now document case studies to motivate the form of the model of consumption and exchange we develop and analyze below.

Exchange of food has occurred across different cultures, $3,5,9,37$ and continues to the present day, for example through market systems of trade. ${ }^{49,50}$ While we will focus here on the material benefits of food exchange, the implications of our model will also raise questions for broader social phenomena, continuing a long history of studying the intertwining of the economics with the social and political contexts of exchange. ${ }^{51}$ Exchange is really a broad set of distinct processes, encompassing food sharing within a household, to internal exchange between individuals within a social or geographic unit, and external trade between individuals or groups in distinct geographic locations. Moreover, sometimes the same frameworks and terminology have been used to describe exchange across these scales-for example, redistribution has been documented among sedentary populations in distinct locations in the Pacific Northwest, ${ }^{3}$ but the same pooling and redistribution terminology is also applied when food is shared immediately among a group of hunters, i.e. on a much smaller spatial and temporal scale.

In this manuscript we will focus on exchange over relatively long distances between populations farming crops-distances that are sufficiently large that exchange of food can potentially buffer temporal variability in yield. This means that variability must be at least somewhat uncorrelated across large enough spatial scales, but that these scales must be small enough that food exchange is plausible (and would not cost more than its benefits). As such, our model most likely applies to marginal and volatile farming environments, where there are significant opportunities to benefit from food exchange to other locations-for example for maize agriculture in Pueblo society in the American Southwest. There is evidence establishing that maize was likely exchanged on length-scales of up to $100 \mathrm{~km}^{14-17}$ among Pueblo communities, while variability in yield across space ${ }^{13}$ has been reconstructed from tree-ring data and estimated to vary on length-scales of $10 \mathrm{~s}$ of $\mathrm{km}$. Finally, biophysical estimates (based on energy expenditure for transportation) have also put the maximum length-scale over which it would have been plausible to exchange maize in this system at around $50 \mathrm{~km},{ }^{44}$ meaning that exchange is a plausible mechanism via which ancestral groups in this region buffered uncertainty. 
Evidence for long-distance exchange as a mechanism to mitigate uncertainty has also been documented for other ancestral societies, for example in agricultural production in Mississipian culture, ${ }^{52}$ and huntergatherer populations bridging mainland California and the Santa Barbara channel islands. ${ }^{53}$ Putting all of this together, it seems likely that food exchange has occurred in multiple contexts, and could often have played a role in buffering weather-related uncertainty, in line with the fourth component of the general framework outlined above. ${ }^{37}$ What is less clear are the precise mechanisms and networks of exchange in ancestral societies. ${ }^{17}$ The evidence above doesn't establish for any particular society who gave to whom, how regular or consistent this exchange was, or how extensively it was practiced. These are for the most part unknowns, although there is certainly strong evidence that crop failure was a regular occurrence in the Southwest, suggesting that some degree of consistency and organization would be plausible, ${ }^{13,44}$ and it has also been proposed that the distribution of food would have been in proportion to surplus. ${ }^{44,54}$ Beyond these motivations, we will keep our modeling framework as flexible as possible, in order to draw the most general conclusions we can. At the same time we will also make some specific choices of functional forms for the consumption and exchange rates of food, in order to generate some understanding of the consequences of food exchange. These will include both the expected consequences in terms of mitigating uncertainty, and also some unexpected consequences, in terms of resource-mediated population instabilities.

\subsection{Model of Consumption and Exchange of Food across Microenvironments}

We now introduce a model human consumers and a single type of food, each at one of $m$ total locations, labeled by $i$. The current standing resource pool at location $i$ is $R_{i}$, and the number of human consumers is $N_{i}$, with their dynamics over time given by:

$$
\begin{aligned}
\frac{d R_{i}}{d t} & =\rho_{i}-c R_{i} N_{i}+\sum_{j} P_{i j} N_{j}-N_{i} \sum_{j} P_{j i} \\
\frac{d N_{i}}{d t} & =c N_{i} R_{i}-\mu N_{i} .
\end{aligned}
$$

The per capita mortality rate of consumers in the absence of food is $\mu$, and consumers eat food at a rate $c R_{i} N_{i}$. We incorporate production of food via $\rho_{i}$, and exchange of resources between location $i$ and location $j$ via a matrix $P_{i j}$, shown schematically in Figure 1. The specific modeling choices we've made for production, consumption and exchange are motivated by the ethnographic studies cited above, and we now discuss these choices (and alternatives) in more detail.

First, the parameters $\rho_{i}$ characterize the environmental context at location $i$, representing the 'inflow' of resources available to use at location $i$. Our model is based on the idea that a given location $i$ will have a carrying capacity (the size of the agricultural niche), and will produce this amount of food each growing season. This amount of food produced over time $T$ is then $\rho_{i} \times T$, or just $\rho_{i}$ if $T$ is taken to be a single year. While agricultural productivity will clearly depend on the activities of the population ${ }^{55}$ at location $i$, there is also a point beyond which additional farmers won't appreciably increase productivity. I.e. there is inevitably an effect of diminishing returns, at which point the largest determinant of productivity is the space and climate at a given location. A more general approach would be to allow resource inflow, $\eta\left(N_{i}\right)$ to be a saturating function of $N_{i}$. For example:

$$
\begin{array}{ll}
\eta\left(N_{i}\right)=\frac{\rho_{i}}{N_{\text {thresh }}} N_{i} & \text { for } N_{i}<N_{\text {thresh }} \\
\eta\left(N_{i}\right)=\rho_{i} & \text { for } N_{i} \geq N_{\text {thresh }}
\end{array}
$$

so that a given location has increasing returns on farming productivity for population size up to a certain threshold, and none beyond that. However, if we restrict populations to land where the productivity that 
each individual can extract from the land is greater than the per capita mortality rate $\left(\frac{\rho_{i}}{N_{\text {thres }}}>\mu\right)$, then in the absence of exchange either $R_{i}$ must be non-decreasing, or $N_{i}$ must be non-decreasing, or both, and eventually the population will not only survive, but continue to grow. This will continue until $N_{i}=N_{\text {thresh }}$ or beyond. After this point, our analysis of equilibrium solutions will reduce to our simpler model given by Eq. (1), and so we proceed using this approach. Finally, we note that Eq. (1) is also a natural approximation to resources that will tend to increase even in the absence of human populations (e.g. fruits).

We next consider consumption in these equations, which occurs via a process proportional to $R_{i} N_{i}$, with constant of proportionality $c$ representing the per capita consumption rate per unit of food, a rate which here is common to all populations. Hence, consumers are only able to draw from the resource pool at their spatial location-i.e. resources at location $j$ are only available to population $i$ when $i=j$. We can also see that overall consumption at location $i$ increases with both number of individuals and with available resources at $i$. This functional form is unlikely to hold across all values of $R_{i}$ and $N_{i}$, but we propose that the basic directionalities of those changes in consumption rates are reasonable-it would also be straightforward to modify this model so that consumption proceeds at a rate $N_{i} C\left(R_{i}\right)$, for an arbitrary function of $R_{i}$, with similar downstream results for stability. In summary, consumption thus both depletes resources, and allows for the maintenance and possible growth of the human population.

Finally, exchange of resources is mediated by the term $P_{i j} N_{j}$ describing the transfer of food from location $j$ to location $i$. We note that this is a particular form of transfer, over large scales-and hence we would not expect that our model should apply to sharing between people who are living in very close proximity to each other. We also note that this transfer process only affects the availability of resources, and does not directly affect population sizes or involve the movement of individuals from site to site. This can be seen by the absence of $P_{i j}$ from the second equation in (1), describing changes in population size. We assume that $P_{i i}=0$, meaning that there is no 'exchange' from a site to itself. Hence resources at location $i$ are depleted only in proportion to $N_{i}$, and supplemented in proportion to each of the other population sizes $N_{j}$, accounting for both terms on the right-hand side of the equation describing $d R_{i} / d t$. Hence, we can think of this exchange process as each population picking up from their own pile of food, and transporting that chunk of food into the pile available to another population. This also means that if there are no individuals at location $j$, then there is no transfer of resources to any other location $i$, and transfer of resources increases in proportion to $N_{j}$, as a kind of per capita tax.

This exchange process is an agreed-upon rate of transfer that always takes place, no matter what the local environmental context, and in proportion to population size at each 'source' location. We will see below that in the absence of exchange, larger equilibrium population sizes precisely correspond to more productive locations (e.g. due to more conducive local climate or simply larger physical size). Viewed in that light our assumption is in essence that locations which produce more resources are (all other things being equal) expected to exchange more resources-we think of the exchange process as built into what populations plan to do with their crop yield. Despite, this, it would clearly be reasonable to introduce more complex functional dependencies of the transfer process on $R_{i}$ and $N_{j}$. In our Supplementary Information we introduce two more general models of exchange, allowing fluctuations up and down in $R_{i}$ to increase or reduce transfer rates, and also allowing for population sizes $N_{i}$ to have a greater or lesser impact on transfer rates. Our primary analytical results in the main body of this manuscript will correspond to the case Eq. (1), but as we detail our results we will also find that similar outcomes for transitions to instability hold even when exchange takes this more general form in terms of dependence on $R_{i}$ and $N_{i}$. These outcomes include both additional anaytical results and numerical results, and are detailed in our Supplementary Information Section 2 (for linear dependence of transfer out of location $i$ on $N_{i}$, and arbitrary dependence on $R_{i}$ ) and Supplementary Information Section 3 (more general dependence on $N_{i}$, including no explicit dependence on population size). 


\section{Results}

\subsection{Exchange can buffer environmental variation}

We now suppose that each microenvironment $i$ experiences changes in $\rho_{i}$ over time, corresponding to variability in the growth rate of food over time. We might expect that exchange of a staple resource will buffer the effects of this environmental variation, so that scarce periods at a given location are buffered by a flow in from outside. Before testing this intuition, we first note that there are equilibrium solutions for this model of the form

$$
\begin{aligned}
R_{i}^{*} & =\mu / c \\
N_{i}^{*} & =\frac{1}{\mu} \sum_{j} M_{i j}^{-1} \rho_{j}
\end{aligned}
$$

where the matrix $M_{i j}=\delta_{i j}+\delta_{i j} \frac{1}{\mu} \sum_{k} P_{k i}-\frac{1}{\mu} P_{i j}$ and $\delta_{i j}$ is the Kronecker delta symbol, $=1$ when $i=j$ and zero otherwise. In our methods section we show that $M^{-1}$ is a left-stochastic matrix, ${ }^{56}$ meaning that it has all entries non-negative, and all column sums equal to 1 . This is sufficient for population sizes $N_{i}^{*}$ to all be positive, given positive local food production rates $\rho_{j}$. In Supplementary Information Section 1, we give a step-by-step derivation of these equilibrium solutions and the subsequent results below on mitigating environmental variability.

Next, we allow the production at each site, $\rho_{i}$, to vary over time. In particular, we assume that all of these production rates are fixed for a given interval of time, and that after this interval concludes each $\rho_{i}$ is re-drawn from a probability distribution. At the beginning of each time interval each population will thus tend to be away from its (new) equilibrium value, and so local populations will tend to increase or decrease corresponding to the new level of production. We suppose that this time-interval is long enough for populations to reach new equilibria, and in this sense the environmental changes here are much slower than the population changes in response. Throughout this process, we assume that to a first approximation per capita consumption rate $c$, mortality rates $\mu$ and exchange rates $P_{i j}$ will be unchanged, so that the only parameters varying are the $\rho_{i}$.

We will initially assume that changes are completely uncorrelated across microenvironments, so that $\left\langle\rho_{i} \rho_{j}\right\rangle=$ $\left\langle\rho_{i}\right\rangle\left\langle\rho_{j}\right\rangle$, where the angled brackets refer to the means and (co-)variances of $\rho_{i}$, taken across time intervals. Even with this assumption, there remains an outstanding question of exactly what distribution underlies variability in agricultural productivity. Here, we use two lines of evidence leading to a relationship between the variance in productivity at a given site, and the mean productivity at that same site. To motivate this, we note that there is a long history in ecology of searching for patterns relating means and variances of population sizes. These are known collectively as Taylor's law, ${ }^{39,57,58}$ which states that for a population of size $n$, empirically we will tend to find that

$$
\operatorname{var}(n) \sim\langle n\rangle^{z}
$$

where $\mathrm{z}$ is an exponent, often in the range $0-2$. In recent work, the temporal version of Taylor's law has been shown to apply at the level of national crop yields (for multiple crops). I.e. the mean and variance (taken over time) of yield follows Taylor's law, with an exponent $z$ close to but less than one. ${ }^{40}$ Moreover, this scaling is consistent with the temporal variability in potential productivity using reconstructions of the maize-farming niche over time in the Southwest ${ }^{2}{ }^{13}$-a second line of evidence for the same phenomenon.

With this motivation in mind, we choose Taylor's law as a flexible and plausible assumption. I.e., we specify that the variance across time of the stochastic variable $\rho_{i}$ is proportional to its mean raised to power $z$ : 


$$
\operatorname{var}\left(\rho_{i}\right)=\beta\left\langle\rho_{i}\right\rangle^{z},
$$

where $\beta$ is a constant across all sites in the region.

We now consider the limiting case of no exchange, as a point of comparison. In this case $P_{i j}=0$ and so we have that the equilibrium population size is proportional to the inflow of resources, $\rho_{i}$, in each location. Specifically, the equilibrium is $N_{i}^{b}=\rho_{i} / \mu$, where the superscript $b$ indicates that this gives us our baseline case. Hence the variance in population size over time is approximately:

$$
\operatorname{var}\left(N_{i}^{b}\right)=\frac{\beta}{\mu^{2-z}}\left\langle N_{i}^{b}\right\rangle^{z} .
$$

In contrast, when $P_{i j}$ is non-zero, we will find new equilibrium solutions for population sizes, which we label $N_{i}^{*}$, as in Eq (3). The means and variances of these population sizes over time are related as follows:

$$
\begin{aligned}
\frac{\operatorname{var}\left(N_{i}^{*}\right)}{\left\langle N_{i}^{*}\right\rangle^{z}} & =\frac{1}{\mu^{2-z}} \frac{\sum_{j} M_{i j}^{-1} M_{i j}^{-1} \operatorname{var}\left(\rho_{j}\right)}{\left(\sum_{k} M_{i k}^{-1}\left\langle\rho_{k}\right\rangle\right)^{z}} \\
& =\frac{\beta}{\mu^{2-z}} \frac{\sum_{j} M_{i j}^{-1} M_{i j}^{-1}\left\langle\rho_{j}\right\rangle^{z}}{\left(\sum_{k} M_{i k}^{-1}\left\langle\rho_{k}\right\rangle\right)^{z}}
\end{aligned}
$$

If $1 \leq z \leq 2$ then

$$
\begin{aligned}
\frac{\operatorname{var}\left(N_{i}^{*}\right)}{\left\langle N_{i}^{*}\right\rangle^{z}} & \leq \frac{\beta}{\mu^{2-z}}\left(\max \left(M_{i j}^{-1}\right)\right)^{2-z} \\
& \leq \frac{\operatorname{var}\left(N_{i}^{b}\right)}{\left\langle N_{i}^{b}\right\rangle^{z}}
\end{aligned}
$$

where $\max \left(M_{i j}^{-1}\right)$ is the largest entry in the matrix $M^{-1}$. The first inequality uses that $z \geq 1$, and the second uses both that $M^{-1}$ is a left-stochastic matrix (i.e. its largest entry is always less than or equal to 1 ), and that $z \leq 2$. In other words, for $1 \leq z \leq 2$, any exchange buffers uncorrelated environmental variance across microenvironments, for every single population. For $0<z<1$, the outcome depends on the specific set of mean $\left\langle\rho_{i}\right\rangle$ and values of $M_{i j}$. However, for a fixed set of $\left\langle\rho_{i}\right\rangle$, sufficently small $\max \left(M_{i j}^{-1}\right)$ will also guarantee that exchange buffers environmental variability. Finally, for $z>2$, a reduction in $\max \left(M_{i j}^{-1}\right)$, i.e. making exchange more extensive, will often tend to increase population variability. In Figure 2 we provide an example of the buffering effect, where we choose a range of strengths for the exchange process. To provide an example, we draw the $\rho_{i}$ from a negative binomial distribution, parametrized in such a way that the variance in each site is proportional to the mean $(z=1)$, with the same fixed $\beta$ across all sites.

\subsection{Only Specific Modes of Exchange Maintain Stability under System-wide Scarcity}

We now derive criteria for the stability of the equilibrium (3). By stability here we mean stable to small perturbations away from the equilibrium solution ${ }^{59}$ (for example an influx or departure of resources or people), a concept that has been used as a criterion for equilibria in economics and finance, ${ }^{60-65}$ and ecology, ${ }^{66,67}$ including ecological systems with explicit resource exchange. ${ }^{28}$ Given that such perturbations are inevitable, we are essentially identifying criteria for whether a given equilibrium solution can ever be seen in a real system of exchange. 
First, we note that the Jacobian matrix $L$ (corresponding to the linearized system near the equilibrium) is a block matrix:

$$
L=\left[\begin{array}{c|c}
-c \operatorname{diag}\left(N_{i}^{*}\right) & -\mu M \\
\hline c \operatorname{diag}\left(N_{i}^{*}\right) & 0
\end{array}\right]
$$

where again $M_{i j}=\delta_{i j}+\delta_{i j} \frac{1}{\mu} \sum_{k} P_{k i}-\frac{1}{\mu} P_{i j}$, and $\operatorname{diag}\left(v_{i}\right)$ indicates the matrix formed from placing the elements of vector $v_{i}$ along the diagonal, and zeroes elsewhere. The equilibrium solution (3) will only be stable to local perturbations if all eigenvalues of $L$ have negative real parts.

To make some progress on deriving criteria for the exchange matrix $P$ that will lead to stable equilibria, we first consider the case where all $N_{i}^{*}=N^{*}$, i.e. all microenvironments have an equal population size. (While this analysis may be reasonable for systems where settlements do not vary considerably in size, we will later relax this assumption and allow for arbitrary $N_{i}^{*}$.) In our methods section, we show that a necessary and sufficient condition for stability is that the following hold for every eigenvalue $\gamma$ of $M$ :

$$
\operatorname{Im}(\gamma)^{2}<\frac{c N^{*}}{\mu} \operatorname{Re}(\gamma)
$$

From the form of $M$ (see methods), plus Gershgorin's theorem, ${ }^{68}$ the real parts of every $\gamma$ must be positive. This indicates that if $c N^{*} / \mu>\max \frac{\operatorname{lm}(\gamma)^{2}}{\operatorname{Re}(\gamma)}$, taken over all $\gamma$, then the equilibrium will be stable.

To explore the consequences of this, we consider what happens when there is correlated variation in all $\rho_{i}$. l.e. we suppose that initially resource inflows are given by $\rho_{i}^{0}$, and then at some point in time all resource inflows are rescaled by a factor $\alpha$, so that new values of $\rho_{i}$ are given by

$$
\rho_{i}=\alpha \rho_{i}^{0} .
$$

Following this change in resource inflow by the overall factor $\alpha$, populations will initially be away from their new equilibrium values, which are

$$
N^{*}=\alpha N^{0 *}
$$

where $N^{0 *}$ was the original equilibrium population size at each site under the initial resource inflow. Hence the necessary and sufficient condition for stability of the new equilibrium $N^{*}$ becomes:

$$
\operatorname{Im}(\gamma)^{2}<\frac{\alpha c N^{0 *}}{\mu} \operatorname{Re}(\gamma)
$$

For sufficiently large values of $\alpha$, it is clear that we can now guarantee satisfying $\frac{\alpha c N^{0 *}}{\mu}>\max \frac{\operatorname{Im}(\gamma)^{2}}{\operatorname{Re}(\gamma)}$. This implies that any given mode of exchange will have a stable equilibrium with positive population sizes, for large enough resource rescaling factors, $\alpha$. In contrast, for sufficiently small $\alpha$, only modes of exchange such that $\operatorname{Im}(\gamma)=0$ for all $\gamma$ will lead to a positive, locally stable equilibrium. Thus, if all eigenvalues of $M$ are real, stability is guaranteed for all values of $\alpha$. But any more generic mode of exchange, where one or more eigenvalue of $M$ has an imaginary part, will be unstable to small perturbations for small enough $\alpha$. We call these cases non-stabilizing, since stability is not guaranteed. We illustrate this transition in Figure 3 , and a similar transition for our more general model in Supplementary Information Sections 2 and 3.

\subsection{Categorizing Stable Modes of Exchange}

For our model, we have assumed equally-sized local populations, and used this to derive a necessary and sufficient condition for stability across all rescalings of resource supply rates, expressed in terms of the 
eigenvalues of the matrix $M$. This necessary and sufficient condition is highly restrictive-for example, the probability that a random matrix has all real eigenvalues falls exponentially with the square of the number of rows of the matrix. ${ }^{69}$ Correspondingly, the chance that a randomly-chosen system of exchange in our model will result in a stable equilibrium in times of scarcity is extremely small, once there are more than a handful of locations.

On the other hand, stating that the eigenvalues of $M$ must be real to ensure stability doesn't give much of a sense of the corresponding structure of $P$. To our knowledge there is no simple, exhaustive classification of the form of non-negative matrices $P$ such that the corresponding matrix $M$ will have only real eigenvalues. However, we can consider several examples, also illustrated in Figure 4:

(i) Altruism. $P_{i j}=0$ for $i<j$. Upper triangular $P$ have real eigenvalues (in fact, all equal to zero since all $P_{i i}=0$ in our model), and the corresponding $M$ are also upper triangular. We can intepret this case as altruism, where there is only unidirectional transfer from any one location to any other. Such exchange roughly corresponds to Sahlins' 'Generalized Reciprocity' [3, Chapter 5], with examples in real populations sometimes falling close to this limit, but also including various degrees of exchange, hospitality, or gift-making.

(ii) Balanced Reciprocity. Symmetric $P$ such that $P_{i j}=P_{j i}$ for all $i$ and $j$ have real eigenvalues, as do the corresponding matrices $M$. We can interpret this case as balanced reciprocity between all groups, so that each population gives the same per capita as it receives from each other population. This symmetric condition seems intuitively 'fair', but on the other hand runs somewhat against the received wisdom in ecological stability, where there is no special role of symmetry in promoting stability in mutualistic interactions. ${ }^{70}$

(iii) Pooling. $P_{i j}=P_{j i}=0$ unless $j=1$ (or any other specific index), with entries $P_{i 1}, P_{1 j}$ arbitrary. This case represents pooling of resources to one central hub, and redistribution from that hub to each other population. I.e. all locations both give to and receive from a single focal location, falling into Sahlins' third category of exchange. In our methods section we prove (along with case (iv)) that this results in a stable equilibrium for all rescaling of resource inflows. We note that even if the population size of the hub is small, transfer out of the hub could be relatively large if $P_{i 1}$ are correspondingly larger than $P_{1 i}$.

(iv) Decentralized Redistribution. We now add a category that unifies and extends categories (i) and (iii). Both of these cases are special cases of the general rule that the exchange network has no directed cycles of length greater than or equal to 3 . I.e. that $P_{i_{1} i_{2}} P_{i_{2} i_{3}} \ldots P_{i_{n}, i_{1}}=0$ for all sets of distinct indices $i_{k}$, for $n \geq 3$. While this category includes altruism, and pooling to a central hub, it also allows for more general extended networks, while not requiring that the exchange be balanced between each pair of populations, as in (ii). Since there is this potentially unequal flow of resources, coupled with the absence of a central hub, we term this decentralized redistribution. In our methods section we show this acyclic property leads to a stable equilibrium.

This list of possible structures is not exhaustive. For example, any similarity transformation of one of the above $M$ matrices resulting in another matrix of the same form (with negative off-diagonal elements and rows that sum to zero) will have identical eigenvalues, and hence also lead to stable exchange. So in that sense, our necessary and sufficient condition stated above in terms of the eigenvalues of $M$ is the cleanest description of stable networks of exchange. On the other hand, it is notable that these examples of exchange matrices $P$ that guarantee stability include Sahlins' classification of exchange between groups, proving that they are among the limited set of exchange networks in our model that will guarantee stability under arbitrary environmental conditions. 


\subsection{Balanced Reciprocity Implies Stability More Generally}

These results are reminiscent of results for stability for Lotka-Volterra-type interactions developed in economics and ecology, where populations interact directly. ${ }^{66,71-74}$ In these models, exchange between two populations would be approximated as a direct effect of one population on the other, without modeling food production, consumption and exhange. As in our model, equilibria in such systems is guaranteed if interactions are acyclic. On the other hand, balanced reciprocity is a stability criterion that only comes to light when exchange is modeled explicitly, as in our model above.

We now relax the assumption that local equilibrium population sizes are equal, and show that reciprocity remains a sufficient condition for stability. In this case, the eigenvalues $\lambda$ of the linearized system near the equilibrium (given by (3)) must solve:

$$
\operatorname{det}\left(\lambda\left(c N_{d}^{*}+\lambda\right) I+\mu c M N_{d}^{*}\right)=0
$$

where $N_{d}^{*}=\operatorname{diag}\left(N_{i}^{*}\right)$ is a shorthand for the diagonal matrix we introduced earlier. In this more general case it is no longer straightforward to solve for $\lambda$ in terms of the eigenvalues of $M$, as we could when all $N_{i}^{*}$ were equal, and so it is not clear what is the corresponding necessary and sufficient condition for stability in this more general case. On the other hand we can still proceed on a case by case basis in terms of establishing explicit examples of stabilizing modes of exchange. In our Methods section we provide an example of this apporach, proving that if $M N_{d}^{*}$ is symmetric, the equilibrium in (3) will be stable.

How should we interpret this condition, now that we have the more general case of unequal $N_{i}^{*}$ ? First, we note that symmetric $M N_{d}^{*}$ provides a sufficient condition for stability under all re-scalings of resource inflow, but it is not a necessary condition. I.e. there could be many other modes of exchange that will guarantee stability. An open question is whether and which more general forms of exchange will guarantee stability, and whether acyclic exchange networks are among these. Our sufficient condition here retains something of the intuition of balanced reciprocity (i.e. there is a specific matrix which needs to be symmetric). But it is no longer true that $P$ itself must be in general be symmetric in order to satisfy this sufficient condition.

In our Supplementary Information we generalize our model in a different way, by allowing for more general dependence of rates of food transfer out of location $i$ on standing resource pools, $R_{i}$, and population sizes $N_{i}$. We provide analytical evidence in SI Section 2 that balanced reciprocity again guarantees stability, even in cases where we allow for an arbitrary dependence of exchange on $R_{i}$ (maintaining also the linear dependence of exchange on $N_{i}$ ), so long as increasing $R_{i}$ tends to increase, rather than decrease transfer out of location $i$. In SI Section 3 we provide numerical evidence that balanced reciprocity also leads to stability when transfer has a more general dependence on both $R_{i}$ and $N_{i}$. Putting all of these results together, we have clear evidence that balanced reciprocity is a stabilizing mode of exchange in somewhat general circumstances.

\subsection{Acyclic Exchange Guarantees Stability under Default for Populations of Equal Size}

Above we established two features of the model of resource exchange characterized by Eq. 1, and illustrated in Figure 1. First, any mode of exchange can potentially reduce the variances in population sizes that arise from uncorrelated fluctuations in resource supply in each microenvironment (Figure 2). On the other hand, correlated changes in resource supply will not only tend to reduce population sizes overall, they can also destabilize the equilibrium solution in Eq. 1, meaning that depopulation may occur even when the equilibrium population sizes are finite, exemplified in Figure 3.

We now consider what happens when $P$ changes over time. For example, we might expect that in periods of scarcity, not all groups will choose to continue to transfer resources at the same rate. If these changes 
are not extremely finely tuned, it's then possible that even our examples of $P$ that guarantee stability may be shifted to new modes of exchange where stability is no longer guaranteed. If we have a matrix $P$ that leads to a stable equilibrium, but this stability property is not robust under renegotiation, the outcome could still lead to depopulation. With this in mind, we now identify a specific scheme under which $P$ might change, and then identify a necessary and sufficient criterion for matrices $P$ that will guarantee stability under this change.

We consider first a fixed $P_{i j}$ and then suppose that one or more of these $P_{i j}$ is instantaneously reduced to a lower (or zero) rate. The idea of this change would be to capture what might happen if a given local population decides to default on its previous commitment to transfer food to one or more other populations. After this change, one or more population sizes will be away from equilibrium. We now consider what will happen to their new equilibrium values, assuming the special case where the initial equilibrium population sizes are equal to each other and the final population sizes are also all equal (but likely different from the initial populations). We know already that stability under scarcity (i.e. when resource inflows are rescaled by a factor $\alpha$, which is then made arbitrarily small) requires that the eigenvalues of $M_{i j}=\delta_{i j}+\delta_{i j} \frac{1}{\mu} \sum_{k} P_{k i}-$ $\frac{1}{\mu} P_{i j}$ are real. In our Methods section we prove that both a sufficient and necessary condition for stability of Eq 1 is that $P$ has no closed loops of non-zero entries, with non-repeated indices, of length greater than two. I.e. $P_{i_{1} i_{2}} P_{i_{2} i_{3}} \ldots . P_{i_{n}, i_{1}}=0$ for all sets of distinct indices $i_{k}$, for $n \geq 3$.

\section{Discussion}

For a model based on the processes of consumption and exchange, we found that exchange of food between different locations could buffer the effects of local variation in productivity. On the other hand, only a limited subset of possible exchange networks guarantees stability when resources become globally scarce. Specifically we showed that when environmental variation is correlated across locations, non-stabilizing modes of exchange will lead to an instability when productivity is sufficiently low. The precise dynamics arising following a perturbation will vary from case to case, but in numerical examples we found that some population sizes will become extremely small. In general we were agnostic about how a particular mode of exchange may have arisen, ${ }^{75-77}$ and what incentives maintain it. ${ }^{78}$ For example, it could be that nonextractive modes of exchange, with no net winners or losers, are in some sense more likely to evolve, or to be evolutionarily stable. But even among this subset of possible modes of exchange, we find examples (e.g. balanced reciprocity) that guarantee stability under all resource inflow rates, and examples (e.g. the ring-like structure in Figure 3) which do not. The fact that the stabilizing modes of exchange that avoid this outcome include (and extend) the classic empirical classifications of exchange made by Sahlins ${ }^{3}$ suggests that stability under a range of environmental conditions is an important criterion in maintaining a given mode of exchange, once it is established.

Several caveats have to be taken into account in considering the implications of our results, including the specific model of exchange, which in its simplest form allows only for constant per capita rates of transfer. We also explored generalizations of this model in our Supplementary Information, introducing a dependence on 'excess' resources, ${ }^{54}$ and a more general dependence of exchange on current population sizes. However, we found that these models showed both analytically and numerically similar results, and in particular we were able to provide evidence that 'reciprocity implies stability' is a robust result across essentially every version of these models we considered. We also show numerical evidence that resource scarcity tends to be destabilizing for more general, non-stabilizing modes of exchange in these more general models, suggesting that the potential resource-mediated instabilities may also be a robust outcome in systems of exchange. For one particular case shown in our Supplementary Information, where exchange is independent of local population sizes, we found that all modes of exchange lead to stable equilibria, but that exchange did not mitigate environmental variability in this case.

A second caveat is that we treated spatial location implicitly, and assumed that exchange is costless. Clearly 
for a spatially-explicit model, the cost of exchange across distances, and the benefits of lack of environmental correlations across those same large distances, may lead to important tradeoffs that are absent here. ${ }^{44}$ Incorporating them may lead to an even richer set of outcomes, or may simply act together to set a preferred distance-scale for settlement separation. A third important issue is that we focused on 'positive' interactions mediated by food exchange. Sahlins' original typology incorporated the possibility of negative reciprocity, i.e. the attempt to get something for nothing. This process would still involve positive rates of transfer, but it seems likely that the rates themselves would be much less likely to be consistent over time than in an agreement designed or evolved to mitigate local environmental fluctuations, and the functional dependencies of transfer on population sizes and resource pools would likely be different from our models-for example the probability of transfer would presumably depend on the size of the recipient population. Even so, it may be that some of our results could still hold for this more general type of interaction.

Despite these caveats, our approach provides a slightly different perspective on the appearance and maintenance of cooperation in human societies, and demonstrates a central message: both resource scarcity and network structure combine to determine stability. For example, earlier studies have clearly shown that exchange modes involving e.g. ring-like transfers can evolve over time, given reasonable models of the evolutionary process..$^{79}$ Our results are entirely compatible with the evolution and persistence of these modes. But our analysis does suggest that the persistence of non-stabilizing modes of exchange will depend on the environmental context: in times of system-wide scarcity, most network structures will tend to lead to unstable dynamics. How could this idea be tested? Long-distance exchange has been established as one among multiple strategies to mitigate uncertainty, ${ }^{37}$ and while many of our results seem quite robust, actually testing which of the quantitative assumptions of our model apply or fail to apply in a given ancestral society will be challenging. An alternative approach may be to establish whether periods of drought or scarcity in climate and productiivity reconstructions tend to correlate with large population fluctuations, ${ }^{1,31,32,80}$ in contrast to steady population decline (without large fluctuations) during these periods. The reality will likely be complicated by other population processes, and a reasonable starting point may be to incorporate the alternative strategies of caloric diversification, human mobility, food storage, and negative interactions, ${ }^{37}$ alongside the exchange processes considered here, to determine whether and under what circumstances we should still predict a signature of instability in times of scarcity. Finally, this interaction of environmental context and network structure has implications for related, resource-limited complex systems, from microbial communities ${ }^{27-29}$ to market-based trade during famine. ${ }^{49,50}$ This potential generality opens up multiple other avenues in which to test how exchange and environment combine to influence stability. 


\section{A Methods}

\section{A.1 Properties of the matrix $M$}

M takes the form $M=I-Q$ where $I$ is the identity matrix, and $Q$ is a matrix with components $Q_{i j}=$ $\frac{1}{\mu} P_{i j}-\delta_{i j} \frac{1}{\mu} \sum_{k} P_{k i}$. I.e. the off-diagonal entries of $M$ are negative and proportional to the off-diagonal entries in the exchange matrix $P$, while the diagonal entries are all positive and larger in magnitude than the column sums of the off-diagonal elements. Using the latter, and Gershgorin's theorem, ${ }^{68}$ the real parts of the eigenvalues of $M$ must be positive. A matrix with negative off-diagonal elements, and whose eigenvalues have non-negative real parts is termed an M-matrix, ${ }^{81}$ and has the property that its inverse, $M^{-1}$, is guaranteed to have non-negative entries. Finally, since $M M^{-1}=I$, and the column sums of $M$ are all equal to one, we can also deduce that the column sums of $M^{-1}$ are equal to one. A non-negative matrix with column sums equal to one is also called a left-stochastic matrix, ${ }^{56}$ and as a corollary each entry must be less than or equal to one.

\section{A.2 Results for Equal Population Sizes}

\section{A necessary and sufficient condition for stability}

Many of our results rest on cases where equilibrium population sizes $N_{i}^{*}$ are all equal. A natural question is whether this condition places constraints on $P_{i j}$. We first note that if all population sizes are equal, then

$$
\rho_{i}=\mu \sum_{j} M_{i j} N_{j}^{*}=\mu N^{*} \sum_{j} M_{i j}
$$

which imposes the following relationship between $P_{i j}$ and $\rho_{i}$ :

$$
\sum_{j} P_{i j}-\sum_{k} P_{k i}=\mu-\frac{1}{N^{*}} \rho_{i}
$$

In essence, so long as $\mu$ is larger than the largest difference between row and column sums of $P$, then we can always find a set of positive $\rho_{i}$ that will lead to equal $N_{i}^{*}$. So these cases are not hard to obtain, but it is fair to say that $\rho_{i}$ and $\mu$ must be somewhat fine-tuned-this is not surprising since clearly it is possible in our model for populations to have unequal population sizes.

Assuming we satisfy this assumption and thus have equal population sizes $N^{*}$, and using standard expressions for the determinant of block matrix form, we can deduce that eigenvalues of $L, \lambda$, must satisfy

$$
\operatorname{det}\left(\lambda\left(c N^{*}+\lambda\right) I+\left(\mu c N^{*}\right) M\right)=0
$$

where $I$ is the identity matrix. We note that this is highly similar to the characteristic equation for eigenvalues $\gamma$ of $M$ itself, which would read:

$$
\operatorname{det}(-\gamma I+M)=0
$$

This means that any $\lambda$ can be related to an eigenvalue of $M$, via the equation:

$$
\lambda^{2}+c N^{*} \lambda+\mu c N^{*} \gamma=0
$$

for some eigenvalue $\gamma$ of $M$. Looking at it the other way round, for every $\gamma$ there are then two solutions for eigenvalues $\lambda$ of $L$, given by solving this quadratic equation to obtain: 


$$
\lambda=\frac{c N^{*}}{2}\left(-1 \pm \sqrt{1-\frac{4 \gamma \mu}{c N^{*}}}\right) .
$$

So in this range of cases where all population sizes are equal, the problem of whether the equilibrium population sizes and resource abundances are stable reduces to identifying specific properties of the eigenvalues of $M$, which in turn depends on the exchange matrix, $P$.

We can now state a necessary and sufficient condition for the real parts of every eigenvalue of $L$ be negative. The Routh-Hurwicz stability criterion states that for real-valued $a_{0}$ and $b_{0}$ the roots of the polynomial

$$
\lambda^{2}+a_{1} \lambda+a_{0}+i b_{0}=0
$$

have negative real part iff both of the following conditions are satisfied:

$$
\begin{aligned}
a_{1} & >0 \\
a_{1}^{2} a_{0} & >b_{0}^{2} .
\end{aligned}
$$

Since both $c$ and $N^{*}$ must be positive numbers for us, the first condition is automatically satisfied. The second condition then translates into the following necessary and sufficient condition for all eigenvalues of $M$ :

$$
\operatorname{Im}(\gamma)^{2}<\frac{c N^{*}}{\mu} \operatorname{Re}(\gamma)
$$

\section{Decentralized networks of exchange are stable for equal population sizes}

We note first that if $P_{i_{1} i_{2}} P_{i_{2} i_{3} \ldots .} \ldots P_{i_{n}, i_{1}}=0$ for all sets of distinct indices $i_{k}$, for $n \geq 3$, then the same is true of the matrix $M$. Next, we note that solving for the eigenvalues $\gamma$ of $M$ is equivalent to solving for the zeros of the characteristic polynomial:

$$
\phi(\gamma, M)=\operatorname{det}(\gamma I-M)=0 .
$$

The characteristic polynomial of an $M$ with no such cycles can be written in a recursive form by removing a single row/column (label $i$ ) at a time: ${ }^{82,83}$

$$
\phi(\gamma, M)=\left(\gamma-M_{i i}\right) \phi\left(\gamma, M^{-(i)}\right)-\sum_{j \neq i} M_{i j} M_{j i} \phi\left(\gamma, M^{-(i, j)}\right)
$$

where $M^{-(i)}$ is constructed from the matrix $M$, but with the $i$-th row and the $i$-th column removed, while $M^{-(i, j)}$ is $M$ with two rows and columns removed. The form of this recursion only involves products of off-diagonal terms of the form $M_{i j} M_{j i}$, and both entries $M_{i j}$ and $M_{j i}$ are guaranteed to be negative for all $i \neq j$, since off-diagonal elements of $P$ must be positive for a reasonable interpretation of the model. Putting these two facts together means that we can always write down a symmetric matrix, $S$ with an identical characteristic polynomial to that of $M$. This symmetric matrix has the same diagonal elements as $M$, and each off-diagonal element is

$$
S_{i j}=S_{j i}=\sqrt{M_{i j} M_{j i}} .
$$

Finally, since $S$ is symmetric, its eigenvalues must be real. Hence $M$ of this form is also guaranteed to have real eigenvalues (because these eigenvalues solve the same equation as those of $S$ ), and so systems with decentralized redistribution are guaranteed to be stable. 


\section{Decentralized networks are necessary for stability when defaults are allowed}

Above, we showed that when all population sizes are equal, a sufficient condition for stability of Eq 1 is that $P$ has no closed loops of non-zero entries, with non-repeated indices, of length greater than two. l.e. $P_{i_{1} i_{2}} P_{i_{2} i_{3} \ldots .} \ldots P_{i_{n}, i_{1}}=0$ for all sets of distinct indices $i_{k}$, for $n \geq 3$. Conversely, suppose there is a closed loop of $k \geq 3$ entries in $P$ such that $P_{i_{1} i_{2}} P_{i_{2} i_{3}} \ldots P_{i_{n}, i_{1}} \neq 0$. Also suppose that arbitrary downward renegotiations are possible. In this case, we test for stability by shrinking every entry of $P$ to zero except $P_{i_{1} i_{2}}, P_{i_{2} i_{3}}, \ldots, P_{i_{n}, i_{1}}$, and we can shrink those remaining entries to the minumum of their values, i.e. the smallest initial value among the set $\left\{P_{i_{1} i_{2}}, P_{i_{2} i_{3}}, \ldots, P_{i_{n}, i_{1}}\right\}$, which we'll just call $p$. By relabelling locations appropriately (corresponding to a permutation of rows and columns in $P$, but not changing the mode of exchange), we can always put this renegotiated $P$ in the block form:

$$
P=\left(\begin{array}{c|c}
\mathcal{P}_{k} & 0 \\
\hline 0 & 0
\end{array}\right)
$$

where the $k \times k$ submatrix $\mathcal{P}_{k}$ is given by

$$
\mathcal{P}_{k}=p\left(\begin{array}{cccc}
0 & 0 & \ldots 0 & 1 \\
1 & 0 & \ldots 0 & 0 \\
0 & 1 & \ldots 0 & 0 \\
\vdots & & & \\
0 & 0 & \ldots 1 & 0
\end{array}\right)
$$

i.e. exchange among the $k$ locations indexed in $\mathcal{P}_{k}$ takes the form of a kind of Kula ring. ${ }^{84,85} \mathcal{P}_{k}$ is a circulant matrix and its eigenvalues $\gamma^{\prime}$ are given ${ }^{86}$ by

$$
\gamma_{j}^{\prime}=p e^{\frac{2 \pi i j(k-1)}{k}} \quad j=0, \ldots, k-1 .
$$

By similar construction the corresponding eigenvalues $\gamma$ of the matrix $M$ are

$$
\gamma=\{\underbrace{1,1+p\left(1-e^{\frac{2 \pi i(k-1)}{k}}\right), 1+p\left(1-e^{\frac{4 \pi i(k-1)}{k}}\right) \ldots, 1+p\left(1-e^{\frac{2 \pi i(k-1)(k-1)}{k}}\right)}_{k \text { values }}, \underbrace{1,1, \ldots, 1}_{(m-k) \text { values }}\}
$$

Since $k \geq 3$, at least two of these eigenvalues will be complex, and hence the renegotiated mode of exchange will be vulnerable to instability for sufficiently scarce resource supply, due to the stability criteria explored in the previous subsection. (This Kula ring-type renegotiation is a kind of worse case scenario, but it is also possible for less extreme renegotiations with closed loops to also lead to complex eigenvalues $\gamma$ ). Finally, we note that this is a highly schematic description of default and renogatiation, and the precise pattern and sensitivity of negotation to environmental conditions will likely play a critical role in any real system. However, what we have shown is that if a set of populations are allowed to arbitrarily reduce nonzero entries in $P_{i j}$, then the equilibrium solution corresponding to this new $P_{i j}$ will be stable if and only if the original exchange network was acyclic.

\section{B Results for arbitrary population sizes}

In this section, we want to show that eigenvalues $\lambda$ of the linearized system, $L$, near equilibrium must have negative real part when $M N_{d}^{*}$ is symmetric, even if $N_{d}^{*}$ is not proportional to the identity. The $\lambda$ must 
therefore satisfy (14) from the main text, derived using a block matrix identity for the determinant of $L$ given in (9):

$$
\operatorname{det}\left(\lambda\left(c N_{d}^{*}+\lambda\right) I+\mu c M N_{d}^{*}\right)=0
$$

First, given that both $N_{d}^{*}$ and $M$ are invertible, the matrix $L$ itself has an inverse, and hence is of full rank. This means that $\lambda \neq 0$. Hence, $\lambda I$ is invertible and we can rewrite the eigenvalue equation as:

$$
\operatorname{det}\left(c N_{d}^{*}+\lambda I+\frac{\mu c}{\lambda} M N_{d}^{*}\right)=0
$$

We now assume that $\operatorname{Re}(\lambda)>0$, and look for a contradiction. The real parts of the eigenvalues of any square matrix lie within the bounds set by the smallest and largest eigenvalues of the Hermitian part of the same matrix [87, Chapter 14]. Hence, if we can show that the Hermitian part of $c N_{d}^{*}+\frac{\mu c}{\lambda} M N_{d}^{*}$ has all positive eigenvalues for any given $\lambda$ with positive real part, then we'll have an inconsistency between this result, the assumption that $\operatorname{Re}(\lambda)>0$, and the eigenvalue equation that each $\lambda$ must satisfy. We now show that the Hermitian part of this matrix is positive definite, and hence has all positive eigenvalues, which will complete the proof by contradiction that symmetric $M N_{d}^{*}$ implies stability.

We first consider the Hermitian part of $\frac{\mu c}{\lambda} M N_{d}^{*}$, which is

$$
H\left(\frac{\mu c}{\lambda} M N_{d}^{*}\right)=\frac{\mu c \operatorname{Re}(\lambda)}{\left|\lambda^{2}\right|} M N_{d}^{*}
$$

where we've used the assumption that $M N_{d}^{*}$ is a symmetric matrix. The matrix multiplication $M N_{d}^{*}$ has the effect of multipling each column of $M$ by a (different) constant, and so we can again use Gershgorin's theorem to show that each eigenvalue of $M N_{d}^{*}$ must be positive. Since it is also symmetric, and since $\mu$, $c$ and $\operatorname{Re}(\lambda)$ and $\left|\lambda^{2}\right|$ are all positive, real numbers, we have that $H\left(\frac{\mu c}{\lambda} M N_{d}^{*}\right)$ is a positive definite matrix. $H\left(c N_{d}^{*}\right)$ is also a symmetric matrix with positive, real eigenvalues (equal to $c N_{i}^{*}$ ), and hence also is positive definite. The sum of two positive definite matrices is also positive definite, and so the Hermitian part of $c N_{d}^{*}+\frac{\mu c}{\lambda} M N_{d}^{*}$ has all positive eigenvalues, completing the proof by contradition: no $\operatorname{Re}(\lambda)$ can be positive, given that $M N_{d}^{*}$ is symmetric. 


\section{Acknowledgments}

We acknowledge helpful comments from multiple colleagues in preparing this manuscript, and the support of Simons Foundation Grant \#376199 and McDonnell Foundation Grant \#220020439.

\section{Author Contributions}

The author contributed all necessary elements for this paper.

\section{Declaration of Interests}

The author declares no competing interests.

\section{Data and Software Availability}

No datasets were generated or analysed during the current study. The author(s) used $\mathrm{R}$ code to generate matrices and simulations necessary to produce Figures 2 and 3, and the SI Figures. The code necessary to generate these figures will be made available in the author's lab github repository, and is also uploaded here.

\section{References}

${ }^{1}$ Mark D Varien, Scott G Ortman, Timothy A Kohler, Donna M Glowacki, and C David Johnson. Historical ecology in the mesa verde region: results from the village ecodynamics project. American Antiquity, 72(2):273-299, 2007.

${ }^{2} \mathrm{R}$ Kyle Bocinsky and Timothy A Kohler. A 2,000-year reconstruction of the rain-fed maize agricultural niche in the us southwest. Nature Communications, 5:5618, 2014.

${ }^{3}$ Marshall Sahlins. Stone age economics. Routledge, 1972.

${ }^{4}$ Elizabeth M Brumfiel, Timothy K Earle, Francoise Audouze, Colin Renfrew, Nathan Schlanger, Andrew Sherratt, Timothy Taylor, Wendy Ashmore, et al. Specialization, exchange and complex societies. Cambridge University Press, 1987.

${ }^{5}$ Michelle Hegmon. Risk reduction and variation in agricultural economies: A computer simulation of Hopi agriculture. 1989.

${ }^{6}$ Bruce Winterhalder. Gifts given, gifts taken: the behavioral ecology of nonmarket, intragroup exchange. Journal of Archaeological Research, 5(2):121-168, 1997.

${ }^{7}$ Bruce Winterhalder and Eric Alden Smith. Analyzing adaptive strategies: human behavioral ecology at twenty-five. Evolutionary Anthropology: Issues, News, and Reviews: Issues, News, and Reviews, 9(2):51-72, 2000.

${ }^{8}$ Michael Gurven. To give and to give not: the behavioral ecology of human food transfers. Behavioral and Brain Sciences, 27(4):543-559, 2004. 
${ }^{9}$ Carolyn D Dillian and Carolyn L White. Introduction: Perspectives on trade and exchange. In Trade and exchange, pages 3-14. Springer, 2010.

${ }^{10}$ Bruce Winterhalder. Diet choice, risk, and food sharing in a stochastic environment. Journal of Anthropological Archaeology, 5(4):369-392, 1986.

${ }^{11} \mathrm{C}$ Athena Aktipis, Lee Cronk, and Rolando de Aguiar. Risk-pooling and herd survival: an agent-based model of a maasai gift-giving system. Human Ecology, 39(2):131-140, 2011.

12 Yan Hao, Dieter Armbruster, Lee Cronk, and C Athena Aktipis. Need-based transfers on a network: a model of risk-pooling in ecologically volatile environments. Evolution and Human Behavior, 36(4):265273, 2015.

${ }^{13}$ R Kyle Bocinsky, Johnathan Rush, Keith W Kintigh, and Timothy A Kohler. Exploration and exploitation in the macrohistory of the pre-hispanic pueblo southwest. Science Advances, 2(4):e1501532, 2016.

${ }^{14}$ Linda S Cordell, Stephen R Durand, Ronald C Antweiler, and Howard E Taylor. Toward linking maize chemistry to archaeological agricultural sites in the north american southwest. Journal of Archaeological Science, 28(5):501-513, 2001.

${ }^{15}$ Larry Benson, Linda Cordell, Kirk Vincent, Howard Taylor, John Stein, G Lang Farmer, and Kiyoto Futa. Ancient maize from chacoan great houses: Where was it grown? Proceedings of the National Academy of Sciences, 100(22):13111-13115, 2003.

${ }^{16}$ Linda S Cordell, H Wolcott Toll, Mollie S Toll, and Thomas C Windes. Archaeological corn from pueblo bonito, chaco canyon, new mexico: dates, contexts, sources. American Antiquity, 73(3):491-511, 2008.

17 Stefani A Crabtree. Inferring ancestral pueblo social networks from simulation in the central mesa verde. Journal of Archaeological Method and Theory, 22(1):144-181, 2015.

${ }^{18}$ Paul Halstead. From reciprocity to redistribution: modelling the exchange of livestock in neolithic greece. Anthropozoologica, 16:19-30, 1992.

${ }^{19}$ Rebecca L Bliege Bird and Douglas W Bird. Delayed reciprocity and tolerated theft: the behavioral ecology of food-sharing strategies. Current anthropology, 38(1):49-78, 1997.

${ }^{20}$ Sean S Downey. Can properties of labor-exchange networks explain the resilience of swidden agriculture? Ecology and Society, 15(4), 2010.

${ }^{21}$ Julia K Clark and Stefani A Crabtree. Examining social adaptations in a volatile landscape in northern mongolia via the agent-based model ger grouper. Land, 4(1):157-181, 2015.

22 Robert MacArthur and Richard Levins. The limiting similarity, convergence, and divergence of coexisting species. American naturalist, pages 377-385, 1967.

${ }^{23}$ David Tilman. Resources: a graphical-mechanistic approach to competition and predation. The American Naturalist, 116(3):362-393, 1980.

${ }^{24}$ Peter Chesson. Macarthur's consumer-resource model. Theoretical Population Biology, 37(1):26-38, 1990.

${ }^{25}$ P. Chesson. Mechanisms of maintenance of species diversity. Annual Review of Ecology and Systematics, 31:343-366, 2000.

${ }^{26}$ Peter A Abrams, Claus Rueffler, and Russell Dinnage. Competition-similarity relationships and the nonlinearity of competitive effects in consumer-resource systems. The American Naturalist, 172(4):463-474, 2008. 
${ }^{27}$ Babak Momeni, Li Xie, and Wenying Shou. Lotka-volterra pairwise modeling fails to capture diverse pairwise microbial interactions. Elife, 6, 2017.

${ }^{28}$ Stacey Butler and James P O'Dwyer. Stability criteria for complex microbial communities. Nature Communications, 9:2970, 2018.

${ }^{29}$ Akshit Goyal, Veronika Dubinkina, and Sergei Maslov. Multiple stable states in microbial communities explained by the stable marriage problem. The ISME journal, page 1, 2018.

${ }^{30}$ Shigeo Yachi and Michel Loreau. Biodiversity and ecosystem productivity in a fluctuating environment: the insurance hypothesis. Proceedings of the National Academy of Sciences, 96(4):1463-1468, 1999.

31 Joseph Tainter. The collapse of complex societies. Cambridge university press, 1990.

${ }^{32}$ Lee Clare and Bernhard Weninger. Social and biophysical vulnerability of prehistoric societies to rapid climate change. Documenta Praehistorica, 37:283-292, 2010.

${ }^{33}$ James H Brown, Joseph R Burger, William R Burnside, Michael Chang, Ana D Davidson, Trevor S Fristoe, Marcus J Hamilton, Sean T Hammond, Astrid Kodric-Brown, Norman Mercado-Silva, et al. Macroecology meets macroeconomics: Resource scarcity and global sustainability. Ecological engineering, 65:24-32, 2014.

${ }^{34}$ Barney T Burns. Simulated Anasazi Storage Behavior Using Crop Yields Reconstructed from Tree Rings, $A D$ 652-1968. PhD thesis, University of Arizona, 1983.

${ }^{35}$ David G Anderson, David W Stahle, and Malcolm K Cleaveland. Paleoclimate and the potential food reserves of mississippian societies: A case study from the savannah river valley. American Antiquity, 60(2):258-286, 1995.

${ }^{36}$ Bruce Winterhalder and Carol Goland. An evolutionary ecology perspective on diet choice. People, plants, and landscapes: Studies in paleoethnobotany, 45879:123, 1997.

${ }^{37}$ Paul Halstead and John O'Shea. Bad year economics: cultural responses to risk and uncertainty. Cambridge University Press, 2004.

${ }^{38}$ Timothy A Kohler, C David Johnson, Mark Varien, Scott Ortman, Robert Reynolds, Ziad Kobti, Jason Cowan, Kenneth Kolm, Schaun Smith, and Lorene Yap. Settlement ecodynamics in the prehispanic central mesa verde region. The model-based archaeology of socionatural systems, pages 61-104, 2007.

39 Joel E Cohen, Meng Xu, and William SF Schuster. Stochastic multiplicative population growth predicts and interprets taylor's power law of fluctuation scaling. Proceedings of the Royal Society B: Biological Sciences, 280(1757):20122955, 2013.

40 Thomas F Döring, Samuel Knapp, and Joel E Cohen. Taylors power law and the stability of crop yields. Field Crops Research, 183:294-302, 2015.

${ }^{41}$ Walter E Baethgen. Climate risk management for adaptation to climate variability and change. Crop Science, 50(Supplement_1):S-70, 2010.

42 David B Lobell, Michael J Roberts, Wolfram Schlenker, Noah Braun, Bertis B Little, Roderick M Rejesus, and Graeme L Hammer. Greater sensitivity to drought accompanies maize yield increase in the us midwest. Science, 344(6183):516-519, 2014.

${ }^{43}$ Alex Wu, Graeme L Hammer, Al Doherty, Susanne von Caemmerer, and Graham D Farquhar. Quantifying impacts of enhancing photosynthesis on crop yield. Nature plants, 5(4):380, 2019.

${ }^{44}$ Kent G Lightfoot. Food redistribution among prehistoric pueblo groups. Kiva, 44(4):319-339, 1979. 
45 John OShea et al. Coping with scarcity: exchange and social storage. Economic archaeology: towards an integration of ecological and social approaches, 96:167-183, 1981.

${ }^{46}$ Cynthia L Herhahn and J Brett Hill. Modeling agricultural production strategies in the northern rio grande valley, new mexico. Human Ecology, 26(3):469-487, 1998.

${ }^{47}$ R Kyle Bocinsky and Mark D Varien. Comparing maize paleoproduction models with experimental data. Journal of ethnobiology, 37(2):282-308, 2017.

${ }^{48}$ Warren R DeBoer. Subterranean storage and the organization of surplus: the view from eastern north america. Southeastern Archaeology, pages 1-20, 1988.

${ }^{49}$ Martin Ravallion et al. Markets and famines. Clarendon Press, 1987.

${ }^{50}$ Martin Ravallion. Famines and economics. journal of Economic Literature, 35(3):1205-1242, 1997.

51 Timothy K Earle. Prehistoric economics and the archaeology of exchange. In Contexts for prehistoric exchange, pages 1-12. Elsevier, 1982.

52 Fred A Finney. Exchange and risk management in the upper mississippi river valley, ad 1000-1200. Midcontinental Journal of Archaeology, pages 353-376, 2000.

53 Jeanne E Arnold. Complex hunter-gatherer-fishers of prehistoric california: Chiefs, specialists, and maritime adaptations of the channel islands. American Antiquity, 57(1):60-84, 1992.

54 Timothy A Kohler and Carla R Van West. The calculus of self-interest in the development of cooperation: sociopolitical development and risk among the northern anasazi. In Evolving complexity and environmental risk in the prehistoric Southwest, pages 169-196. CRC Press, 1996.

55 Timothy A Kohler. Modeling agricultural productivity and farming effort. Emergence and Collapse of Early Villages: Models of Central Mesa Verde Archaeology, pages 85-112, 2012.

${ }^{56}$ Samuel Karlin. A first course in stochastic processes. Academic press, 2014.

${ }^{57}$ LR Taylor. Aggregation, variance and the mean. Nature, 189(4766):732, 1961.

${ }^{58}$ LR Taylor and IP Woiwod. Temporal stability as a density-dependent species characteristic. The Journal of Animal Ecology, pages 209-224, 1980.

${ }^{59}$ Aleksandr Mikhailovich Lyapunov. The general problem of the stability of motion. International journal of control, 55(3):531-534, 1892.

60 Paul A Samuelson. The stability of equilibrium: comparative statics and dynamics. Econometrica: Journal of the Econometric Society, pages 97-120, 1941.

${ }^{61}$ Kenneth $\mathrm{J}$ Arrow and Leonid Hurwicz. On the stability of the competitive equilibrium, i. Econometrica: Journal of the Econometric Society, pages 522-552, 1958.

62 Herbert Scarf. Some examples of global instability of the competitive equilibrium. International Economic Review, 1(3):157-172, 1960.

${ }^{63}$ Franklin M Fisher. The stability of general equilibrium: Results and problems. 1976), Essays in Economic Analysis: Proceedings of the Association of University Teachers of Economics Sheffield, pages 3-29, 1975.

${ }^{64}$ Robert M May, Simon A Levin, and George Sugihara. Complex systems: Ecology for bankers. Nature, 451(7181):893, 2008. 
65 John William Hatfield, Scott Duke Kominers, Alexandru Nichifor, Michael Ostrovsky, and Alexander Westkamp. Stability and competitive equilibrium in trading networks. Journal of Political Economy, 121(5):9661005, 2013.

${ }^{66}$ Robert M May. Will a large complex system be stable? Nature, 238(5364):413-414, 1972.

${ }^{67}$ Crawford S Holling. Resilience and stability of ecological systems. Annual review of ecology and systematics, 4(1):1-23, 1973.

${ }^{68}$ Roger A. Horn and Charles R. Johnson. Matrix Analysis. Cambridge University Press, New York, NY, USA, 2nd edition, 2012.

${ }^{69}$ Alan Edelman. The probability that a random real gaussian matrix haskreal eigenvalues, related distributions, and the circular law. Journal of Multivariate Analysis, 60(2):203-232, 1997.

70 Jordi Bascompte, Pedro Jordano, and Jens M Olesen. Asymmetric coevolutionary networks facilitate biodiversity maintenance. Science, 312(5772):431-433, 2006.

71 James Quirk and Richard Ruppert. Qualitative economics and the stability of equilibrium. The review of economic studies, 32(4):311-326, 1965.

${ }^{72}$ Clark Jeffries. Qualitative stability and digraphs in model ecosystems. Ecology, 55(6):1415-1419, 1974.

${ }^{73}$ Clark Jeffries, Victor Klee, and Pauline Van den Driessche. When is a matrix sign stable. Technical report, WASHINGTON UNIV SEATTLE DEPT OF MATHEMATICS, 1976.

74 Jeffrey M Dambacher, Hang-Kwang Luh, Hiram W Li, and Philippe A Rossignol. Qualitative stability and ambiguity in model ecosystems. The American Naturalist, 161(6):876-888, 2003.

${ }^{75}$ Robert Axelrod and William Donald Hamilton. The evolution of cooperation. Science, 211(4489):13901396, 1981.

${ }^{76}$ Samuel Bowles and Herbert Gintis. Origins of human cooperation. Genetic and cultural evolution of cooperation, 2003:429-43, 2003.

${ }^{77}$ Samuel Bowles and Herbert Gintis. The evolution of strong reciprocity: cooperation in heterogeneous populations. Theoretical population biology, 65(1):17-28, 2004.

${ }^{78}$ Robert Boyd, Herbert Gintis, Samuel Bowles, and Peter J Richerson. The evolution of altruistic punishment. Proceedings of the National Academy of Sciences, 100(6):3531-3535, 2003.

${ }^{79}$ Robert Boyd and Peter J Richerson. The evolution of indirect reciprocity. Social Networks, 11(3):213-236, 1989.

${ }^{80}$ Bernhard Weninger, Lee Clare, Eelco Rohling, Ofer Bar-Yosef, Utz Böhner, Mihael Budja, Manfred Bundschuh, Angelica Feurdean, Hans Georg Gebe, Olaf Jöris, et al. The impact of rapid climate change on prehistoric societies during the holocene in the eastern mediterranean. Documenta praehistorica, 36:7-59, 2009.

${ }^{81}$ Abraham Berman and Robert J Plemmons. Nonnegative matrices in the mathematical sciences, volume 9. Siam, 1994.

${ }^{82}$ Allen J Schwenk. Computing the characteristic polynomial of a graph. In Graphs and combinatorics, pages 153-172. Springer, 1974.

${ }^{83}$ Francesco Belardo, Enzo M Li Marzi, and Slobodan K Simić. Schwenk-like formulas for weighted digraphs. In AIP Conference Proceedings, volume 1168, pages 1251-1254. AIP, 2009. 
84 JP Singh Uberoi. Politics of the Kula ring: an analysis of the findings of Bronislaw Malinowski. Manchester University Press, 1962.

${ }^{85}$ Ronald M Sabatelli and Constance L Shehan. Exchange and resource theories. In Sourcebook of family theories and methods, pages 385-417. Springer, 2009.

${ }^{86}$ Philip J Davis. Circulant matrices. American Mathematical Soc., 2012.

${ }^{87}$ Leslie Hogben. Handbook of linear algebra. Chapman and Hall/CRC, 2006. 


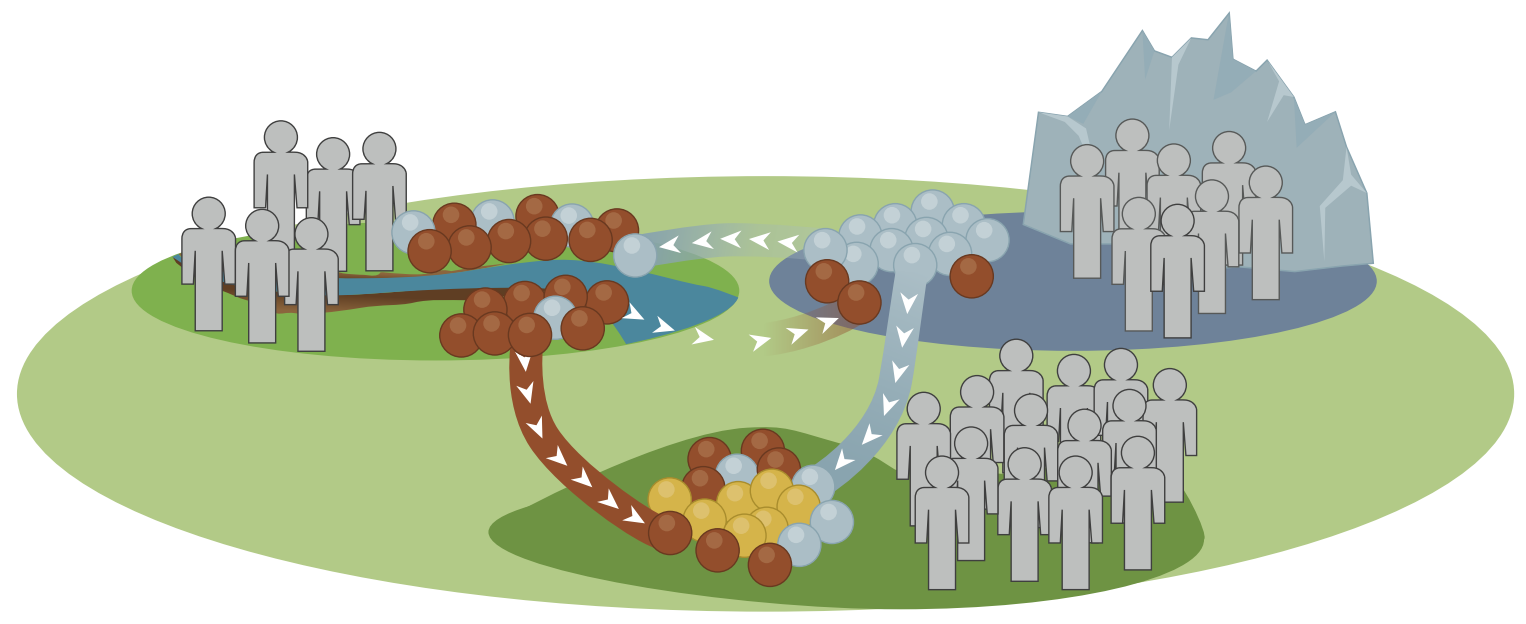

Figure 1: Model of Populations Consuming and Exchanging a Staple Resource. Our model consists of multiple locations, three of which are shown in the figure. Within each location, a human population consumes from resource pools of varying sizes. All resources are identical, but color here indicates the origin of the resource. Each environment has an inflow of new resources characterizing the productivity of the environment, and there is a per capita rate mortality rate for all consumers. Critically, transfer between each habitat is possible indicated by the arrows. 
Population Sizes through time
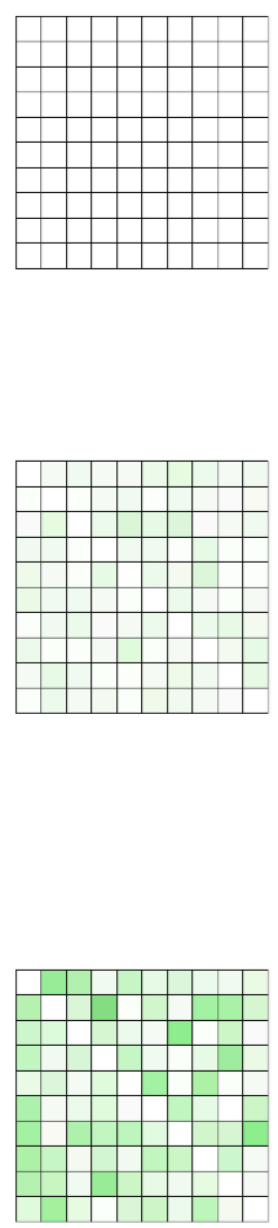

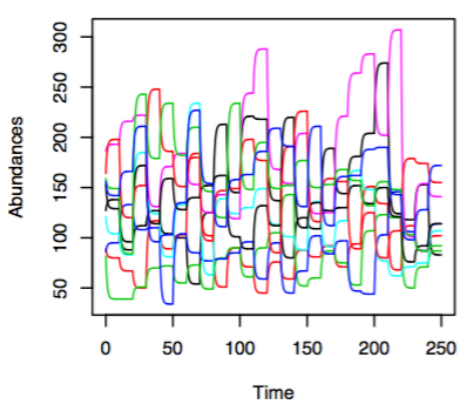

Population Sizes through time

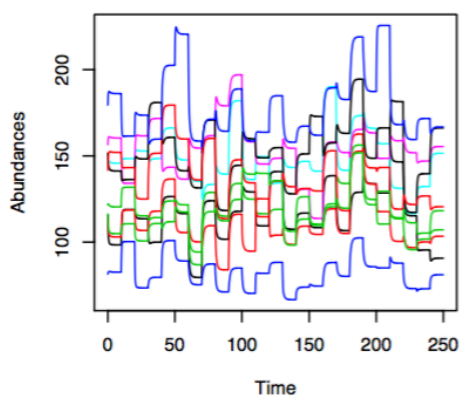

Population Sizes through time

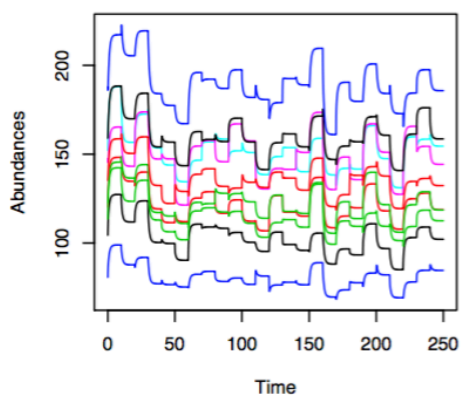

Resource Pools through time

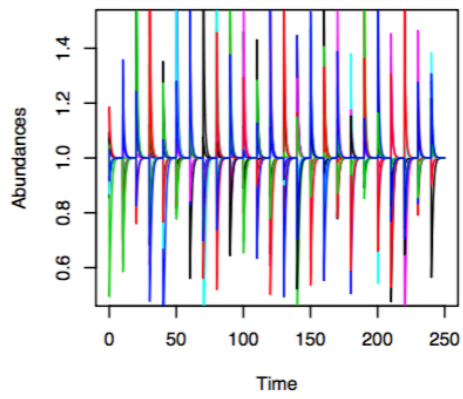

Resource Pools through time

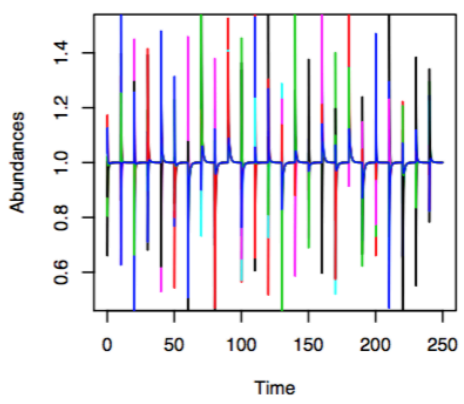

Resource Pools through time

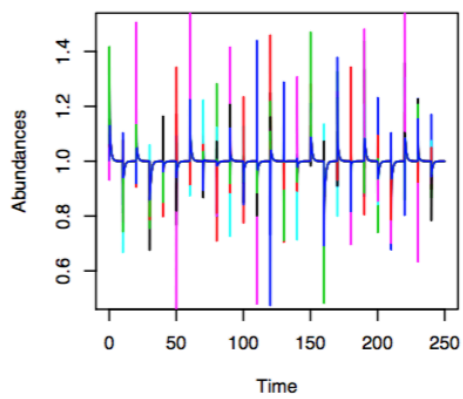

Figure 2: Exchange Buffers Local Variation. In this plot we demonstrate the buffering effect of exchange, which allows a spreading of risk when microenvironment productivies change over time, and are uncorrelated. Here we show three examples of exchange among ten microenvironments. The left hand set of panels shows the exchange matrix $P$, which is zero in the first system, and has non-zero off-diagonals in the next two cases. For both of these, the off-diagonal entries are the absolute values of a variable randomly drawn from a Gaussian distribution with mean zero and variance 1 , and the third case has an overall factor of two in the strength of exchange relative to the second case. In the middle and right hand panels we show the effect of a fluctuating resource inflow $\rho_{i}$, on the dynamics of both population sizes (middle) and resource pools (right), with different colors representing the populations and pools in different environments. The mean value of $\rho_{i}$ (when averaged across time) is drawn from a negative binomial distribution with a given mean and variance, so that some microenvironments are (on average) more productive than others. Then, every $10 / \mu$ timesteps, $\rho_{i}$ in each microenvironment is redrawn from a negative binomial distribution with mean $\rho_{i}$, and variance $10 \rho_{i}$, satisfying the assumptions in (5) with $\beta=10$, and representing a slow time-dependence for local productivity. As strength of exchange increases, variation in population sizes decreases. 

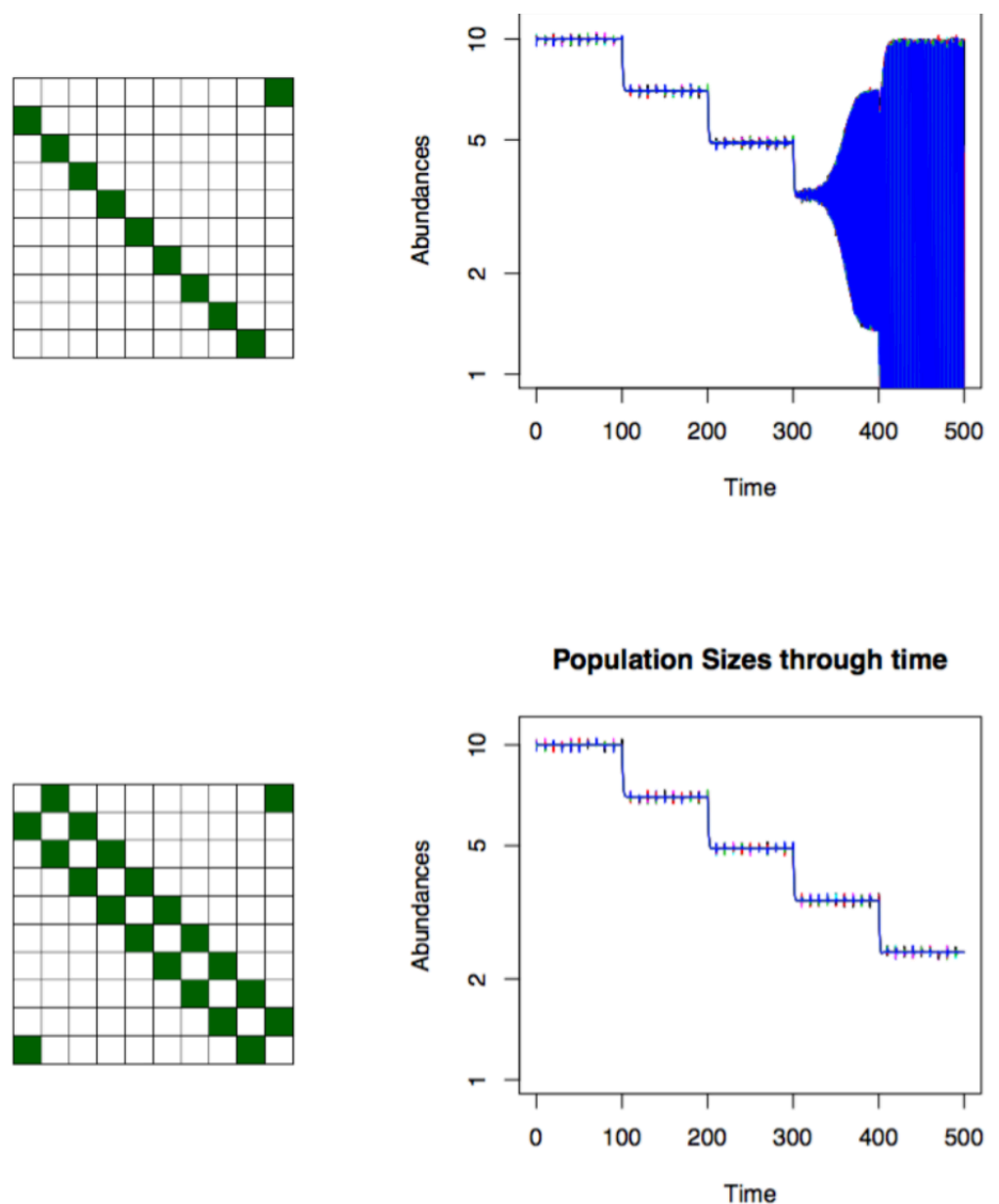

Figure 3: Non-stabilizing modes of exchange lead to instability under system-wide scarcity. In the upper panel, we consider a Kula ring-type mode of exchange, where each microenvironment passes to the next in a kind of loop. ${ }^{84,85}$ Notably, the Kula ring mode of exchange is not found for exchange of food, but here we consider it as a theoreticallypossible mode of exchange because it will (like any other exchange matrix $P$ ) tend to buffer local variation, and is non-extractive. In the lower panel we consider a symmetrized version of the same exchange matrix, so that exchange is exactly reciprocal. For these two cases, we choose $\rho_{i}$ equal for each environment $i$, which for these modes of exchange also leads to equal equilibrium population sizes $N_{i}^{*}$ in each microenvironment. The right hand panels show the dynamics of these populations through time, under correlated, system-wide shifts downward in resource inflow, as might be the case for a prolonged drought or other source of scarcity. We also introduce small perturbations in both populations and resources, and see that initially both upper (Kula) and lower (symmetrized) equilibria are stable to these small perturbations. Each downward shift in $\rho_{i}$ leads to a lower equilibrium population size, but at a critical point the Kula mode of exchange leads to an instability, and small perturbations are amplified. This suggests a reason that such cyclic modes of exchange may be undesirable for food exchange. 


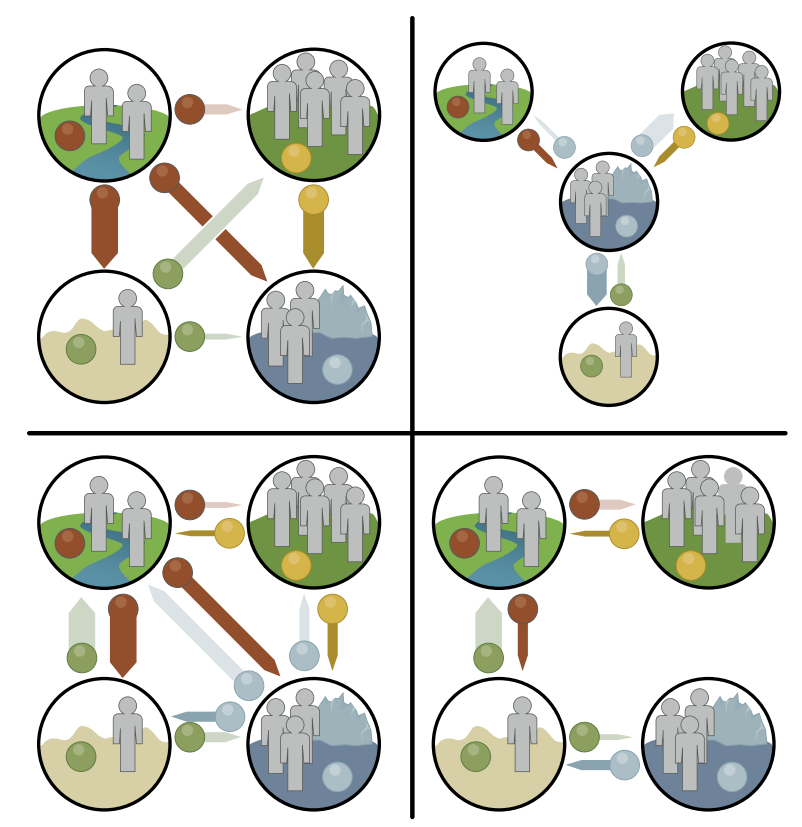

Figure 4: Examples of modes of exchange that guarantee stability under system-wide scarcity. Only particular modes of exchange will guarantee equilibria that are stable when resources are scarce. In this figure we give four classes that are stable, showing a visual representation of an example exchange network in each case. The top left shows pure altruism, where no transfer is reciprocated, and $P$ takes an upper triangular form. Bottom left shows examples of balanced recipricity, where every exchange is exactly reciprocated, at the same rate. Top right shows pooling and redistribution, while the bottom right shows a decentralized network of exchange with no cycles. 


\section{Stability Constrains how Populations Spread Risk in a Model of Food Exchange: Supplementary Information}

\section{More detailed derivations of solutions in the main text}

\subsection{Equilibrium Solutions and their Stability}

We now give a more detailed derivation of solutions to the model described in Eq. (1) of the main text. I.e.: 5

$$
\begin{aligned}
\frac{d R_{i}}{d t} & =\rho_{i}-c R_{i} N_{i}+\sum_{j} P_{i j} N_{j}-N_{i} \sum_{j} P_{j i} \\
\frac{d N_{i}}{d t} & =c N_{i} R_{i}-\mu N_{i} .
\end{aligned}
$$

The equilibrium solutions here must satisfy:

$$
\begin{aligned}
& 0=\rho_{i}-c R_{i}^{*} N_{i}+\sum_{j} P_{i j} N_{j}^{*}-N_{i}^{*} \sum_{j} P_{j i} \\
& 0=c N_{i}^{*} R_{i}^{*}-\mu N_{i}^{*},
\end{aligned}
$$

and assuming that population sizes are non-zero, we can first tackle the second of these two equations, and divide out to eliminate $N_{i}^{*}$. This leaves us with

$$
R_{i}^{*}=\frac{\mu}{c}
$$

Next, we can use this equilibrium solution for each $R_{i}$ and plug it into the population equilibrium equation to find:

$$
0=\rho_{i}-\mu N_{i}+\sum_{j} P_{i j} N_{j}^{*}-N_{i}^{*} \sum_{j} P_{j i} .
$$

This means that the equilibrium solution for population sizes must satisfy:

$$
N_{i}^{*}=\frac{1}{\mu} \sum_{j} M_{i j}^{-1} \rho_{j}
$$

where $M_{i j}=\delta_{i j}+\delta_{i j} \frac{1}{\mu} \sum_{k} P_{k i}-\frac{1}{\mu} P_{i j}, \delta_{i j}$ is the Kronecker delta symbol (=1 when $i=j$ and zero otherwise), 9 and we think of $M_{i j}$ as the components of a matrix. $M_{i j}^{-1}$ are then the components of the inverse matrix 10 $M^{-1}$ that satisfies $M M^{-1}=M^{-1} M=I$. As stated in the methods section of our main text, a matrix $M \quad{ }_{11}$ of this form (given that the entries of $P$ are all non-negative, since they must correspond to non-negative 12 rates of transfer) is actually termed in the literature an M-matrix [1], and has the property that the entries 13 
of its inverse $M^{-1}$ are all positive. This means that the equilibrium solutions for population sizes $N_{i}^{*}$ are (fortunately) positive. Any other outcome would mean that our model was not even plausibly a well-defined description of equilibrium population sizes.

The Jacobian matrix $L$ (corresponding to the linearized system near the equilibrium) is a block matrix:

$$
L=\left[\begin{array}{c|c}
-c \operatorname{diag}\left(N_{i}^{*}\right) & -\mu M \\
\hline c \operatorname{diag}\left(N_{i}^{*}\right) & 0
\end{array}\right]
$$

where $\operatorname{diag}\left(v_{i}\right)$ indicates the matrix formed from placing the elements of vector $v_{i}$ along the diagonal, and zeroes elsewhere. The eigenvalues of $L$ will determine whether the equilibrium solution above is stable - if any eigenvalues have positive real part, then small perturbations will tend to grow, and the equilibrium is classified as unstable. The eigenvalues of $L$ satisfy

$$
\operatorname{det}(L-\lambda I)=0
$$

meaning that we need to compute the determinant of a matrix of block form

$$
Y=\left[\begin{array}{l|l}
A & B \\
\hline C & D
\end{array}\right]
$$

where we can assume that $C$ and $D$ commute, since $D$ is proportional to the identity matrix. If we can solve for this determinant, and then solve the resulting polynomial equation to compute the eigenvalues, $\lambda$, we will hence be able to determine whether any $\lambda$ have positive real part for any given parameter values.

The determinant of a block matrix of this form $Y$ where $C$ and $D$ commute is given by:

$$
\operatorname{det}(Y)=\operatorname{det}(A D-B C)
$$

Hence, when all $N_{i}^{*}$ are equal to the same population size, $N^{*}$, we can deduce that eigenvalues of $L, \lambda$, must satisfy

$$
\operatorname{det}\left(\lambda\left(c N^{*}+\lambda\right) I+\left(\mu c N^{*}\right) M\right)=0
$$

where $I$ is the identity matrix. We note that this is highly similar to the characteristic equation for eigenvalues $\gamma$ of $M$ itself, which would read:

$$
\operatorname{det}(-\gamma I+M)=0
$$


This means that any $\lambda$ can be related to an eigenvalue of $M$, via the equations detailed in our Methods 31 section of the main manuscript. I.e. that

$$
\lambda^{2}+c N^{*} \lambda+\mu c N^{*} \gamma=0
$$

for some eigenvalue $\gamma$ of $M$. Looking at it the other way round, for every $\gamma$ there are then two solutions for ${ }^{33}$ eigenvalues $\lambda$ of $\mathrm{L}$, given by solving this quadratic equation to obtain:

$$
\lambda=\frac{c N^{*}}{2}\left(-1 \pm \sqrt{1-\frac{4 \gamma \mu}{c N^{*}}}\right) .
$$

So in this range of cases where all population sizes are equal, the problem of whether the equilibrium pop- 35 ulation sizes and resource abundances are stable reduces to identifying specific properties of the eigenvalues 36 of $M$, which in turn depends on the exchange matrix, $P$.

We can now state a necessary and sufficient condition for the real parts of every eigenvalue of $L$ be 38 negative. The Routh-Hurwicz stability criterion states that for real-valued $a_{0}$ and $b_{0}$ the roots of the ${ }^{39}$ polynomial

$$
\lambda^{2}+a_{1} \lambda+a_{0}+i b_{0}=0
$$

have negative real part iff both of the following conditions are satisfied:

$$
\begin{gathered}
a_{1}>0 \\
a_{1}^{2} a_{0}>b_{0}^{2} .
\end{gathered}
$$

Since both $c$ and $N^{*}$ must be positive numbers for us, the first condition is automatically satisfied. The ${ }^{42}$ second condition then translates into the following necessary and sufficient condition for all eigenvalues of 43 $M:$

$$
\operatorname{Im}(\gamma)^{2}<\frac{c N^{*}}{\mu} \operatorname{Re}(\gamma)
$$

\subsection{Mitigating Environmental Fluctuations}

In the main text, we used the equilibrium solutions above for the dependence of population sizes on envi- ${ }^{46}$ ronmental parameters $\rho_{j}$ :

$$
N_{i}^{*}=\frac{1}{\mu} \sum_{j} M_{i j}^{-1} \rho_{j}
$$


to determine whether exchange plays a role in mitigating the effect of variability in $\rho_{j}$ on variability over time in $N_{i}^{*}$. I.e. is the effect of food exchange to lessen the variability of population sizes. To determine this, we stated that the variance over time in population sizes would approximately (ignoring transients) be given by:

$$
\frac{\operatorname{var}\left(N_{i}^{*}\right)}{\left\langle N_{i}^{*}\right\rangle^{z}}=\frac{1}{\mu^{2-z}} \frac{\sum_{j} M_{i j}^{-1} M_{i j}^{-1} \operatorname{var}\left(\rho_{j}\right)}{\left(\sum_{k} M_{i k}^{-1}\left\langle\rho_{k}\right\rangle\right)^{z}}
$$

This numerator here uses that

$$
\begin{aligned}
\operatorname{var}\left(N_{i}^{*}\right) & =\left\langle N_{i}^{* 2}\right\rangle-\left\langle N_{i}^{*}\right\rangle^{2} \\
& =\frac{1}{\mu^{2}} \sum_{j} M_{i j}^{-1} \sum_{j^{\prime}} M_{i j^{\prime}}^{-1}\left(\left\langle\rho_{j} \rho_{j^{\prime}}\right\rangle-\left\langle\rho_{i}\right\rangle\left\langle\rho_{j^{\prime}}\right\rangle\right) .
\end{aligned}
$$

If the environmental variability over time is uncorrelated at the different locations, as assumed in this part of the main text, then we can rewrite this last line as:

$$
\begin{aligned}
\frac{1}{\mu^{2}} \sum_{j} M_{i j}^{-1} \sum_{j^{\prime}} M_{i j^{\prime}}^{-1}\left(\left\langle\rho_{j} \rho_{j^{\prime}}\right\rangle-\left\langle\rho_{i}\right\rangle\left\langle\rho_{j^{\prime}}\right\rangle\right) & =\frac{1}{\mu^{2}} \sum_{j} M_{i j}^{-1} \sum_{j^{\prime}} M_{i j^{\prime}}^{-1} \operatorname{var}\left(\rho_{j}\right) \delta_{j j^{\prime}} \\
& =\frac{1}{\mu^{2}} \sum_{j} M_{i j}^{-1} M_{i j}^{-1} \operatorname{var}\left(\rho_{j}\right) .
\end{aligned}
$$

as used above and in the main text Eq.(7).

In the main text, we then used our assumption of Taylor's law with parameter $z$ as a description (justified by empirical data on crop yield fluctuations) of the distribution of $r h o_{j}$ over time at any one location. This meant we could set $\operatorname{var}\left(\rho_{j}\right)$ in the equation above equal to the mean yield raised to power $z$. I.e. Taylor's law here states that $\operatorname{var}\left(\rho_{j}\right) \propto\left\langle\rho_{j}\right\rangle^{z}$. Finally, we identified the following inequality, valid for $1 \leq z \leq 2$ :

$$
\frac{\beta}{\mu^{2-z}} \frac{\sum_{j} M_{i j}^{-1} M_{i j}^{-1}\left\langle\rho_{j}\right\rangle^{z}}{\left(\sum_{k} M_{i k}^{-1}\left\langle\rho_{k}\right\rangle\right)^{z}} \leq \frac{\beta}{\mu^{2-z}}\left(\max \left(M_{i j}^{-1}\right)\right)^{2-z}
$$

which leads to a statement about when and why exchange mitigates environmental variability. To see why this inequality holds, consider the related function

$$
f(x)=\frac{a^{2} x^{z}+b^{2}}{(a x+b)^{z}}
$$

which is essentially the function above for two locations, where we have focused on variability at a single location $i$, setting $M_{i 1}^{-1}=a, M_{i 2}^{-1}=b$, and $x=\frac{\rho_{1}}{\rho_{2}}$, for notational simplicity. We've also dropped the factor 
of $\beta / \mu^{2-z}$ as this is present on both sides of the inequality. First, note that for $z \geq 1$ and $x \geq 0$ (the latter 64 being the only interpretable range of values for the ratio $\rho_{1} / \rho_{2}$ )

$$
(a x+b)^{z} \geq a^{z} x^{z}+b^{z}
$$

This uses e.g. that the two functions are equal when $x=0$, but the left-hand side has a larger positive 66 gradient than the right-hand-side for all non-negative $x$, and hence is always larger than the right hand side 67 for $x>0$. Hence, we can say that

$$
f(x) \leq \frac{a^{2} x^{z}+b^{2}}{a^{z} x^{z}+b^{z}}=g(x)
$$

I.e. this new function $g(x)$ is greater than or equal to $f(x)$ for $z \geq 1$ and $x>0$. Next, we have two 69 limiting cases, occuring when one site is on average infinitely more productive than the other, and vice 70 versa. In these limits, $g(0)=b^{2-z}$, while $\lim _{x \rightarrow \infty} g(x)=a^{2-z}$. Moreover, $g(x)$ is monotonic, so that 71 $\min \left(a^{2-z}, b^{2-z}\right) \leq g(x) \leq \max \left(a^{2-z}, b^{2-z}\right)$. Finally, we impose a second limit on the range of $z$. If $z$ is 72 bounded above by $z=2$, i.e. if we assume that $1 \leq z \leq 2$, and also use the property that all entries of 73 $M^{-1}$ must be between 0 and 1 (described in more detail above and in the main paper), then we can say that 74 $g(x) \leq \max (a, b)^{2-z}$, which is also between 0 and 1 . This leads to the result that

$$
f(x) \leq \max (a, b)^{2-z}
$$

The full result above and in the main text then relies on the extension of this same approach to more than 76 two locations.

\section{Dependence of the Exchange Process on $R_{i}$}

We argued in the main text that it is plausible for transfer rates to depend linearly on $N_{i}$, a proxy for the of location $i$ on the remaining, steady-state resource pool, $R_{i}$, as well as a more general (including little or no) dependence the population size $N_{i}$. We first tackle the potential dependence of transfer on the steady state pool (distinct from the overall productivity of a given location). In our model we found that it is in 83 fact independent of location:

$$
R_{i}^{*}=\mu / c
$$


I.e. productive sites in general lead to larger populations (subject to the details of the exchange process), and unproductive sites lead to smaller populations, but what's 'left over' in our model is the same either way.

For this reason, varying $\rho_{i}$ doesn't affect this equilibrium value of $R_{i}^{*}$ in the same way that it changes $N_{i}^{*}$, but it's possible that fluctuations up and down in $R_{i}$ could act to increase or reduce transfer rates. For that reason, we now explore the more general model:

$$
\begin{aligned}
\frac{d R_{i}}{d t} & =\rho_{i}-c R_{i} N_{i}+\sum_{j} P_{i j} N_{j} f\left(\frac{c R_{j}}{\mu}\right)-N_{i} f\left(\frac{c R_{i}}{\mu}\right) \sum_{j} P_{j i} \\
\frac{d N_{i}}{d t} & =c N_{i} R_{i}-\mu N_{i} .
\end{aligned}
$$

where the function $f(x)$ introduces a dependence of exchange on the standing pool of resources at location $i$. If we define wlog $\mathrm{f}(1)=1$, then this model actually has equilibria solutions identical to our initial model without the function $f$ :

$$
\begin{aligned}
R_{i}^{*} & =\mu / c \\
N_{i}^{*} & =\frac{1}{\mu} \sum_{j} M_{i j}^{-1} \rho_{j}
\end{aligned}
$$

However, the stability of this equilibrium now depends on the new Jacobian,

$$
L=\left[\begin{array}{c|c}
-c \operatorname{diag}\left(N_{i}^{*}\right)-X & -\mu M \\
\hline c \operatorname{diag}\left(N_{i}^{*}\right) & 0
\end{array}\right]
$$

where the components of the matrix $X$ are given by:

$$
X_{i j}=\frac{c f^{\prime}(1)}{\mu}\left[\delta_{i j} N_{i}^{*} \sum_{k} P_{k i}-P_{i j} N_{j}^{*}\right]
$$

using the chain rule, and the notation that $f^{\prime}(1)$ is whatever the derivative of $f(x)$ is with respect to $x$, evaluated at $x=1$.

\subsection{Symmetric, Resource-Dependent Exchange Implies Population Stability}

Since $X$ is not proportional to the identity (or even diagonal) computing the spectrum is significantly more challenging than in the case of exchange independent of $R_{i}$. On the other hand, we can take a similar approach to the case of unequal population sizes (but transfer rates independent of $R_{i}$ ) considered in the main text. In that example, we also could not derive a general result for the eigenvalue spectrum of $L$, but 
what we could do was proceed on a case-by-case basis to test whether a given mode of exchange would lead 102 to guaranteed stable population equilibria. In particular, we proposed that symmetry of transfer would still 103 be an example of a stabilizing mode of exchange, and found that it was. Here, again we cannot compute the 104 eigenvalue spectrum of $L$ exactly, and so we will try the same approach. We'll assume that the matrix $P$ 105 is symmetric, and show analytically that this is incompatible with any eigenvalues of $L$ having positive real 106 part. We'll then go on to show some numerical examples that are consistent with this result (as they must 107 be). For simplicity, in both numerics and analytics we'll return to the case of equal $N_{i}^{*}=N^{*}$. ${ }_{108}$

Defining $\phi=f^{\prime}(1)$, the eigenvalues $\lambda$ of this linearized system (defined by this new $L$ above) must satisfy: $\quad 109$

$$
\begin{aligned}
\operatorname{det}\left[\lambda\left(\lambda I+c N^{*} I+\frac{\phi c}{\mu} \delta_{i j} N^{*} \sum_{k} P_{k i}-\frac{\phi c}{\mu} N^{*} P_{i j}\right)+\mu c N^{*} M\right] & =0 \\
\Rightarrow \operatorname{det}\left[I \lambda+c N^{*} \tilde{M}+\frac{\mu c N^{*} M}{\lambda}\right] & =0
\end{aligned}
$$

where we used that $L$ is of full rank for the second line (since both $M$ and $c N^{*} I$ are of full rank), and defined ${ }_{110}$ the new matrix

$$
\tilde{M}_{i j}=\delta_{i j}+\frac{\phi}{\mu}\left(\delta_{i j} \sum_{k} P_{k i}-P_{i j}\right) .
$$

Much like $M, \tilde{M}$ takes the form $\tilde{M}=I-\tilde{Q}$ where $I$ is the identity matrix, and $\tilde{Q}$ is a matrix with components 112 $\tilde{Q}_{i j}=\frac{\phi}{\mu} P_{i j}-\delta_{i j} \frac{\phi}{\mu} \sum_{k} P_{k i}$. I.e. the off-diagonal entries of $M$ are negative and proportional to the off-diagonal ${ }_{113}$ entries in the exchange matrix $P$, while so long as $\mathrm{f}(\mathrm{x})$ is an increasing function of the standing resource pool ${ }_{114}$ (i.e. exchange rates tend to increase rather than decrease with $R_{i}$ ) the diagonal entries are all positive and 115 larger in magnitude than the column sums of the off-diagonal elements. Using the latter, and Gershgorin's ${ }^{116}$ theorem [2], the real parts of the eigenvalues of $\tilde{M}$ must be positive. We will now assume that $\operatorname{Re}(\lambda)>0$, ${ }_{117}$ and look for a contradiction. The real parts of the eigenvalues of any square matrix lie within the bounds 118 set by the smallest and largest eigenvalues of the Hermitian part of the same matrix [3, Chapter 14]. Hence, 119 if we can show that the Hermitian part of $c N^{*} \tilde{M}+\frac{\mu c N^{*}}{\lambda} M$ has all positive eigenvalues for any given $\lambda$ with ${ }_{120}$ positive real part, then we'll have an inconsistency between this result, the assumption that $\operatorname{Re}(\lambda)>0$, and ${ }^{121}$ the eigenvalue equation that each $\lambda$ must satisfy.

We now show that the Hermitian part of this matrix is positive definite, and hence has all positive ${ }^{123}$ eigenvalues, which will complete the proof by contradiction that symmetric $P$ implies stability. We first ${ }^{124}$ consider the Hermitian part of $\frac{\mu c N^{*}}{\lambda} M$, which is

$$
H\left(\frac{\mu c N^{*}}{\lambda} M\right)=\frac{\mu c N^{*} \operatorname{Re}(\lambda)}{\left|\lambda^{2}\right|} M
$$

where we've used the assumption that $M$ is a symmetric matrix. Since $M$ also has all positive eigenvalues, ${ }^{126}$ 
and since $\mu, c, N^{*}$ and $\operatorname{Re}(\lambda)$ and $\left|\lambda^{2}\right|$ are all positive, real numbers, we have that $H\left(\frac{\mu c N^{*}}{\lambda} M\right)$ is a positive definite matrix. $H\left(c N^{*} \tilde{M}\right)$ is also a symmetric matrix with positive, real eigenvalues and hence also is positive definite. The sum of two positive definite matrices is also positive definite, and so the Hermitian part of $c N^{*} \tilde{M}+\frac{\mu c N^{*}}{\lambda} M$ has all positive eigenvalues, given that $\operatorname{Re}(\lambda)>0$, completing the proof by contradition: no $\operatorname{Re}(\lambda)$ can be positive, given that $P$ is symmetric.

To give some further insight into stability for this model, we solved numerically for trajectories undergoing small perturbations, analogous to Figure 3 of the main text. Specifically, we chose $f(x) \propto x^{y}$ for an exponent, $y>0$ (so that exchange increases with $R_{i}$ ), and we investigated the dynamics as resource inflow is reduced in a stepwise fashion. For the "Kula Ring" type exchange process (as used in Figure 3 of the manuscript) we found that reducing all resources below a threshold would lead to instability. For a range of values of the exponent $y$ we see in SI Fig 1 the same qualitative outcome. When resource inflow is reduced below a threshold, the solutions Eq. (28) becomes unstable. In contrast, by symmetrizing $P$ we don't see any evidence of instability over the same range of inflow rates, supporting the analytical result just above that symmetry implies stability. This evidence provides an indication that resource-mediated instability, as well as the possibility of specific, stabilizing modes of exchange, is a highly robust, general outcome.

\section{More General Dependence of the Exchange Process on both $N_{i}$ and $R_{i}$}

As a final generalization of our model, we now consider exchange dynamics between location $i$ and other locations that depend both on standing resource pool, $R_{i}$, and also on a more general function $g\left(N_{i}\right)$ of population size at location $i$ :

$$
\begin{aligned}
\frac{d R_{i}}{d t} & =\rho_{i}-c R_{i} N_{i}+\sum_{j} P_{i j} g\left(N_{j}\right) f\left(\frac{c R_{j}}{\mu}\right)-g\left(N_{i}\right) f\left(\frac{c R_{i}}{\mu}\right) \sum_{j} P_{j i} \\
\frac{d N_{i}}{d t} & =c N_{i} R_{i}-\mu N_{i} .
\end{aligned}
$$

Identifying equilibrium solutions for $R_{i}$ follows in the same way as all of our previous models, but the equilibrium population sizes will now depend on the specific choices for $g(x)$ made in formulating this more general model. Computing analytical results for this equilibrium (assuming there is one) is correspondingly more difficult to say something so general as in Section 2 above, or in the (even) simpler model in the main manuscript. We take two approaches here. First, we consider the case numerically where $f(x)=x^{y_{1}}($ as in SI Figure 1) and $g(x)=x^{y_{2}}$, for varying values of $y_{1}$ and $y_{2}$, shown in SI Figure 2. In all of these cases we again see numerical evidence for both instability when resource productivity is low, and that symmetric $P$ 
is stabilizing. Second, we go to a limiting case where still $f(x)=x^{y_{1}}$, but now $g(x)=1$, i.e. so that there is 154 now only a dependence of food exchange on standing resource pools, and no dependence on $N_{i}$. ${ }_{155}$

\subsection{Exchange independent of $N_{i}$}

In this limiting case,

$$
\begin{aligned}
\frac{d R_{i}}{d t} & =\rho_{i}-c R_{i} N_{i}+\sum_{j} P_{i j}\left(\frac{c R_{j}}{\mu}\right)^{y_{1}}-\left(\frac{c R_{i}}{\mu}\right)^{y_{1}} \sum_{j} P_{j i} \\
\frac{d N_{i}}{d t} & =c N_{i} R_{i}-\mu N_{i},
\end{aligned}
$$

and it is actually possible to calculate the equilibrium and full spectrum analytically, since

$$
\begin{aligned}
R_{i}^{*} & =\mu / c \\
N_{i}^{*} & =\frac{1}{\mu}\left(\rho_{i}+\sum_{k} P_{i k}-\sum_{k} P_{k i}\right)
\end{aligned}
$$

and

$$
L=\left[\begin{array}{c|c}
-c \operatorname{diag}\left(N_{i}^{*}\right)-Z & -\mu I \\
\hline c \operatorname{diag}\left(N_{i}^{*}\right) & 0
\end{array}\right]
$$

where the components of the matrix $Z$ are given by:

$$
Z_{i j}=\frac{\phi c}{\mu}\left[\delta_{i j} \sum_{k} P_{k i}-P_{i j}\right]
$$

and $\phi=f^{\prime}(1)$ as above. For this block Jacobian $L$, its eigenvalues satisfy:

$$
0=\operatorname{det}(L-\lambda I)=\operatorname{det}\left(\lambda I+\frac{\mu c N^{*}}{\lambda} I+c N^{*} I+Z\right)
$$

which, following the same approach as before, means that we can relate eigenvalues $\lambda$ of $L$ to the eigenvalues $\quad 161$ $\zeta$ of another matrix - this time $\zeta$ are the eigenvalues of $c N^{*} I+Z$. In particular:

$$
\begin{aligned}
\lambda+\frac{\mu c N^{*}}{\lambda} & =-\zeta \\
\Rightarrow \lambda^{2}+\lambda \zeta+\mu c N^{*} & =0
\end{aligned}
$$


In fact, the Routh Hurwitz criterion applied to this quadratic equation tells us that so long as $\mu c N^{*}$ is positive (which it must be for any reasonably interpretation of these parameters), then all $\lambda$ will have negative real part, and this equilibrium will be stable, independent of the eigenvalues of $Z$ and hence independent of the mode of exchange.

This provides us an example of a kind of exchange system where any mode of exchange will avoid instability. On the other hand, there is one clear difference here. Unlike for example the model in SI Section 2 with both $N_{i}$ and $R_{i}$-dependence for the exchange process, the equilibrium solution Eq. (36) tells us that there is no real pooling of risk in this model. The variance of $N_{i}^{*}$ over time at a given location will always be proportional the variance over time in $\rho_{i}$ at that same location, with the cosntant of proportionality $\mu$ independent of the exchange matrix $P$. This suggests an interesting set of next steps for the analysis here. Some systems of exchange (namely, this process where exchange is independent of $N_{i}$ ) do not effectively mitigate the variation in population size due to variability in yield over time. Others (in the main text, and in SI Section 2 above, and in the more general cases in this SI Section) mitigate uncertainty over a broad range of environmental circumstances, but are vulnerable to leading to population instabilities. Whether an exchange system can be designed that both mitigates uncertainty and avoids instability though remains an open question. 

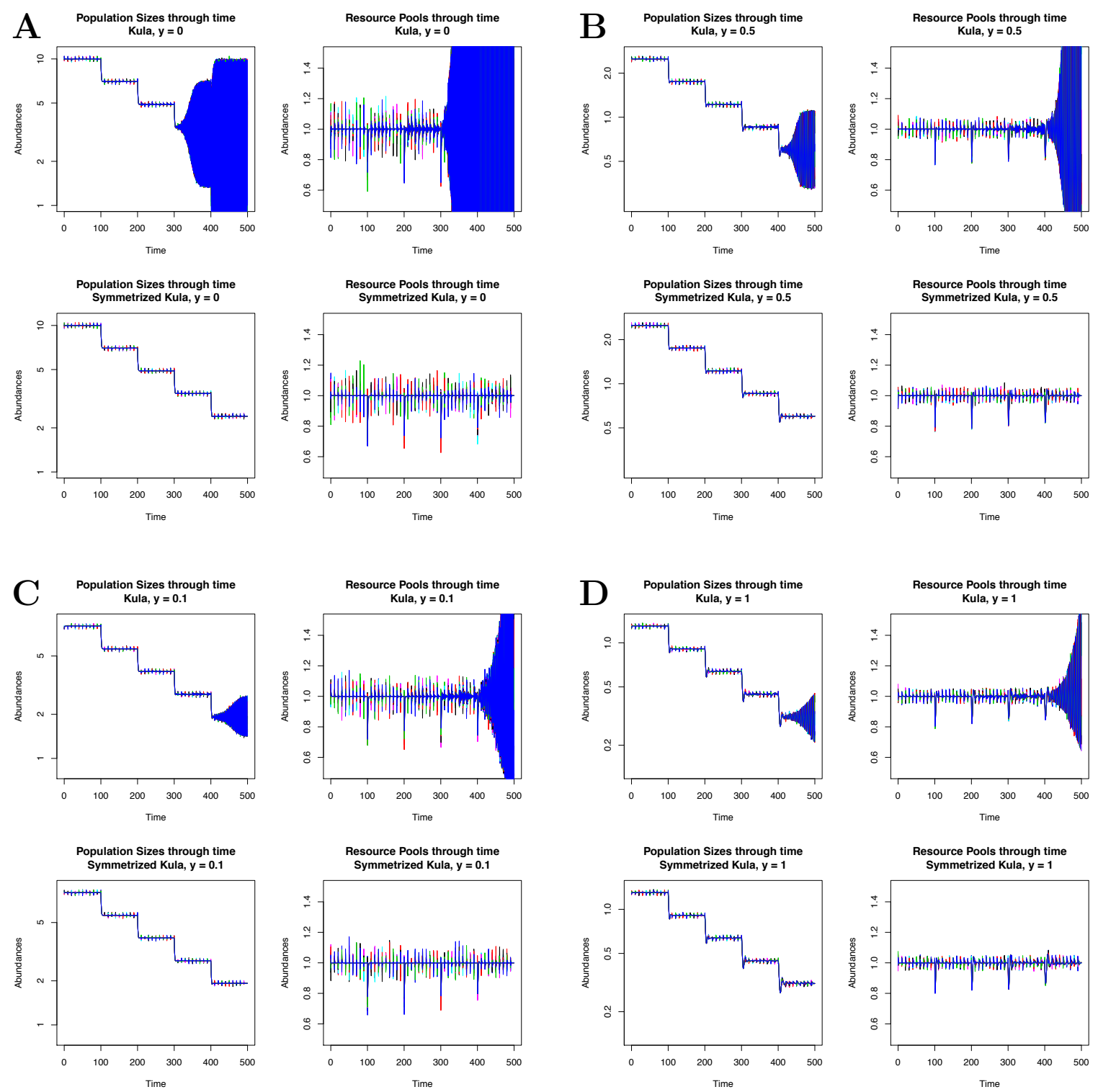

Supplementary Figure 1: Instability/stability under system-wide scarcity for symmetric and asymmetric exchange, with exchange away from a location $i$ proportional to $R_{i}^{y}$ at that location, for various $y$ in panels A-D. The original case shown in our main text and previous draft is $y=0$, shown in Panel A. This case exemplifies the stability results proved in the main text: for the ring-like exchange structure (termed "Kula" in the plot titles) we find that when resource inflow is reduced below a certain level, the equilibrium is unstable. For the symmetrized version of the same exchange process (i.e. where every location now reciprocates with both partners) there is no instability, again consistent with our mathematical proofs. In Panels B-D, we introduce a dependence on local resource pool, $R_{i}$. Our numerical solutions display qualitatively the same features as Panel A: for the ring-like exchange process, the equilibrium solution becomes unstable below a certain threshold of resource inflow, but for the symmetrized exchange process the equilibrium solution is stable for all inflow rates, as proved in the SI text Section 2. 

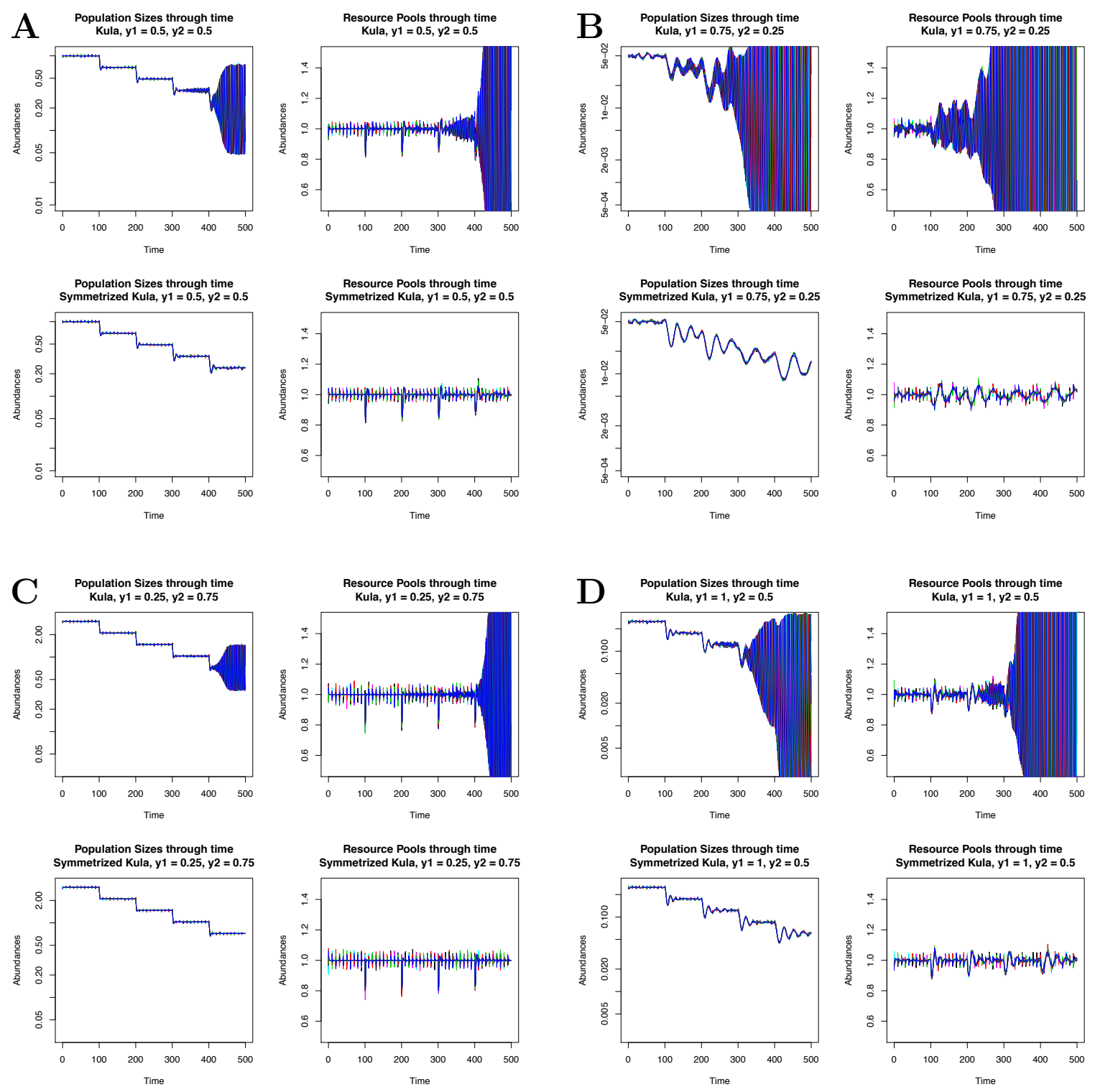

Supplementary Figure 2: Instability/stability under system-wide scarcity for symmetric and asymmetric exchange, with exchange away from a location $i$ proportional to $R_{i}^{y_{1}} N_{i}^{y_{2}}$ at that location, for various $y_{1}$ and $y_{2}$ in panels A-D. For the ring-like exchange structure (termed "Kula" in the plot titles) we find that when resource inflow is reduced below a certain level, the equilibrium is unstable. For the symmetrized version of the same exchange process (i.e. where every location now reciprocates with both partners) there is no instability.

\section{References}

[1] Abraham Berman and Robert J Plemmons. Nonnegative matrices in the mathematical sciences, volume 9. Siam, 1994.

[2] Roger A. Horn and Charles R. Johnson. Matrix Analysis. Cambridge University Press, New York, NY, 
USA, 2nd edition, 2012.

[3] Leslie Hogben. Handbook of linear algebra. Chapman and Hall/CRC, 2006. 